\title{
Basic Searching, Interpolating, and Curve-Fitting Algorithms in $\mathrm{C}++$
}

\author{
by Robert J Yager
}




\section{NOTICES}

\section{Disclaimers}

The findings in this report are not to be construed as an official Department of the Army position unless so designated by other authorized documents.

Citation of manufacturer's or trade names does not constitute an official endorsement or approval of the use thereof.

Destroy this report when it is no longer needed. Do not return it to the originator. 


\title{
Army Research Laboratory
}

Aberdeen Proving Ground, MD 21005-5066

\section{Basic Searching, Interpolating, and Curve-Fitting Algorithms in C++}

\author{
Robert J Yager
}

Weapons and Materials Research Directorate, ARL 


\section{REPORT DOCUMENTATION PAGE}

Public reporting burden for this collection of information is estimated to average 1 hour per response, including the time for reviewing instructions, searching existing data sources, gathering and maintaining the data needed, and completing and reviewing the collection information. Send comments regarding this burden estimate or any other aspect of this collection of information, including suggestions for reducing the burden, to Department of Defense, Washington Headquarters Services, Directorate for Information Operations and Reports (0704-0188), 1215 Jefferson Davis Highway, Suite 1204, Arlington, VA 22202-4302. Respondents should be aware that notwithstanding any other provision of law, no person shall be subject to any penalty for failing to comply with a collection of information if it does not display a currently valid OMB control number.

PLEASE DO NOT RETURN YOUR FORM TO THE ABOVE ADDRESS.

\begin{tabular}{|l|l|l}
\hline 1. REPORT DATE (DD-MM- $Y Y Y Y)$ & 2. REPORT TYPE & 3. DATES COVERED (From - To)
\end{tabular}

\begin{tabular}{l|l} 
January 2015 & Final
\end{tabular}

4. TITLE AND SUBTITLE

January 2014-July 2014

Basic Searching, Interpolating, and Curve-Fitting Algorithms in $\mathrm{C}++$ 5a. CONTRACT NUMBER

\begin{tabular}{|c|c|}
\hline & 5b. GRANT NUMBER \\
\hline & 5c. PROGRAM ELEMENT NUMBER \\
\hline \multirow[t]{2}{*}{$\begin{array}{l}\text { 6. AUTHOR(S) } \\
\text { Robert J Yager }\end{array}$} & $\begin{array}{l}\text { 5d. PROJECT NUMBER } \\
\text { AH80 }\end{array}$ \\
\hline & $\begin{array}{l}\text { 5e. TASK NUMBER } \\
\text { 5f. WORK UNIT NUMBER }\end{array}$ \\
\hline $\begin{array}{l}\text { 7. PERFORMING ORGANIZATION NAME(S) AND ADDRESS(ES) } \\
\text { US Army Research Laboratory } \\
\text { ATTN: RDRL-WML-A } \\
\text { Aberdeen Proving Ground, MD 21005-5066 }\end{array}$ & $\begin{array}{l}\text { 8. PERFORMING ORGANIZATION } \\
\text { REPORT NUMBER } \\
\text { ARL-TN-0657 }\end{array}$ \\
\hline \multirow[t]{2}{*}{ 9. SPONSORING/MONITORING AGENCY NAME(S) AND ADDRESS(ES) } & 10. SPONSOR/MONITOR'S ACRONYM(S) \\
\hline & $\begin{array}{l}\text { 11. SPONSOR/MONITOR'S REPORT } \\
\text { NUMBER(S) }\end{array}$ \\
\hline
\end{tabular}

Approved for public release; distribution is unlimited.

\section{SUPPLEMENTARY NOTES}

\section{ABSTRACT}

This report documents a set of functions, written in $\mathrm{C}++$, that can be used to perform interpolations (nearest-neighbor, linear, and cubic) and to find coefficients for best-fit equations. Functions for working with periodic equations are included.

\section{SUBJECT TERMS}

interpolate, linear, cubic, hermite, polynomial, fit

\begin{tabular}{|l|l|l|l|l|l|}
\hline \multicolumn{2}{|l|}{ 16. SECURITY CLASSIFICATION OF: } & $\begin{array}{l}\text { 17. LIMITATION } \\
\text { OF ABSTRACT }\end{array}$ & $\begin{array}{l}\text { 18. NUMBER } \\
\text { OF PAGES }\end{array}$ & $\begin{array}{l}\text { 19a. NAME OF RESPONSIBLE PERSON } \\
\text { Robert J Yager }\end{array}$ \\
\cline { 1 - 1 } $\begin{array}{l}\text { a. REPORT } \\
\text { Unclassified }\end{array}$ & $\begin{array}{l}\text { b. ABSTRACT } \\
\text { Unclassified }\end{array}$ & $\begin{array}{l}\text { c. THIS PAGE } \\
\text { Unclassified }\end{array}$ & UU & 40 & $\begin{array}{l}\text { 19b. TELEPHONE NUMBER (Include area code) } \\
410-278-6689\end{array}$ \\
\hline
\end{tabular}




\section{Contents}

$\begin{array}{ll}\text { List of Figures } & \text { vi }\end{array}$

Acknowledgments vii

$\begin{array}{lr}\text { 1. Introduction } & 1\end{array}$

2. Background $\quad 1$

3. Searching Sorted Arrays: The BinarySearch() Function 2

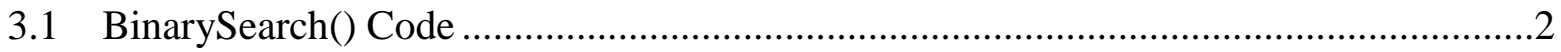

3.2 BinarySearch() Template Class .........................................................................2

3.3 BinarySearch() Parameters ........................................................................................2

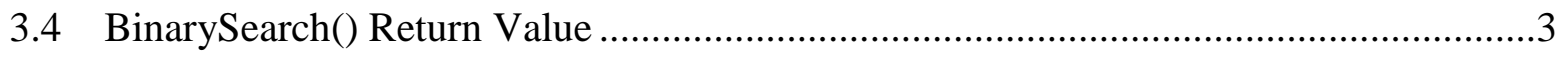

3.5 BinarySearch() Simple Example .....................................................................

3.6 BinarySearch() Text Example ..........................................................................4

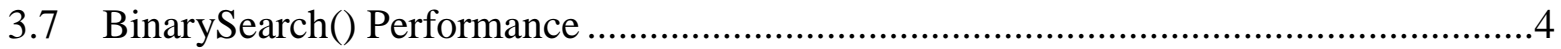

4. Searching Sorted Arrays: The PeriodicSearch() Function 6

4.1 PeriodicSearch() Code................................................................................6

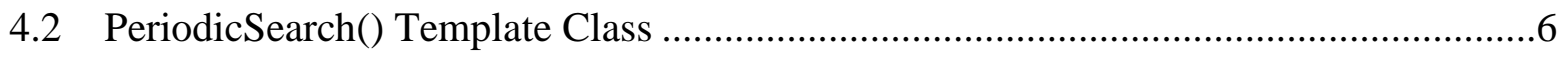

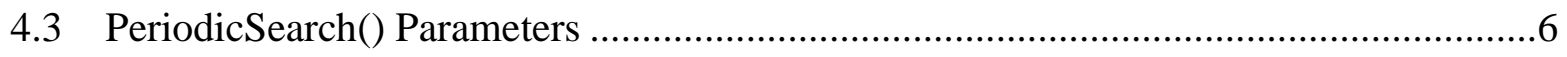

4.4 PeriodicSearch() Return Value.....................................................................

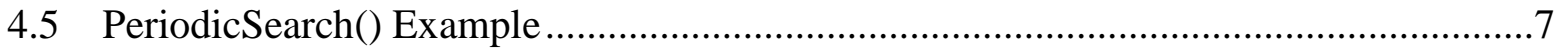

5. Performing Nearest-Neighbor Interpolations: The NNInterp() Function 8

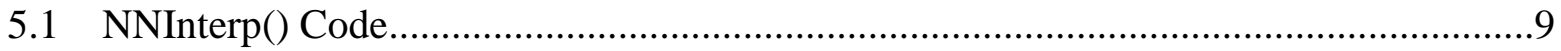

5.2 NNInterp() Template Class ............................................................................ 9

5.3 NNInterp() Parameters ..................................................................................... 9

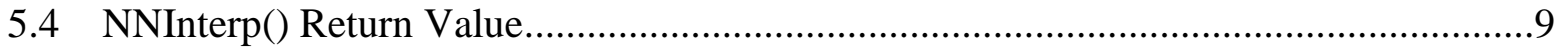

5.5 NNInterp() Simple Example .......................................................................

6. Performing Linear Interpolations: The LinInterp() Function 10 


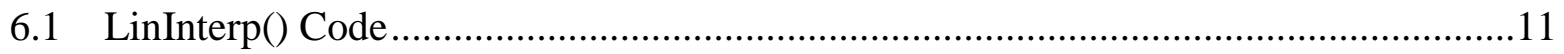

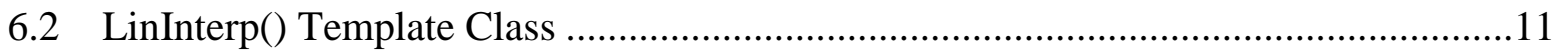

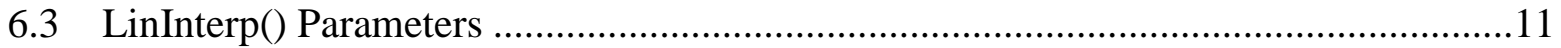

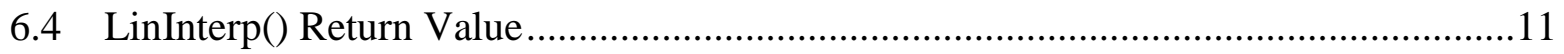

6.5 LinInterp() Simple Example................................................................................11

7. Performing Cubic Interpolations: The CubeInterp() Function 12

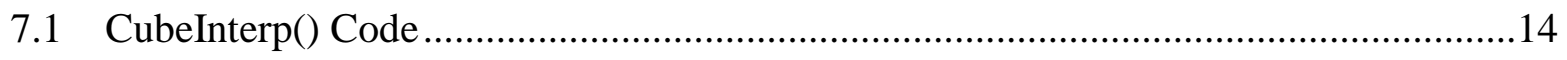

7.2 CubeInterp() Template Class............................................................................14

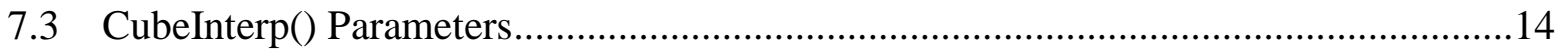

7.4 CubeInterp() Return Value .......................................................................... 14

7.5 CubeInterp() Simple Example ......................................................................14

8. Cardinal Splines: The CardinalSlope() Function 15

8.1 CardinalSlope() Code ......................................................................................15

8.2 CardinalSlope() Template Class............................................................................15

8.3 CardinalSlope() Parameters ............................................................................... 16

8.4 CardinalSlope() Return Value ................................................................................ 16

8.5 CardinalSlope() Simple Example ................................................................ 16

9. Example: Comparing Interpolating Functions 17

10. Example: Performing Periodic Interpolations 17

11. Example: Determining Interpolation Performance $\quad 18$

12. Example: Interpolating in Two Dimensions 19

13. Polynomial Curve Fitting: The PolyFit() Function $\quad 21$

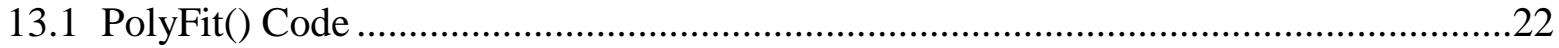

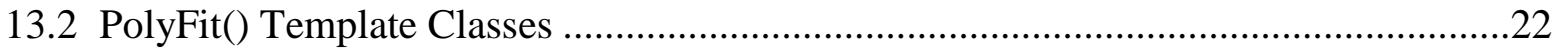

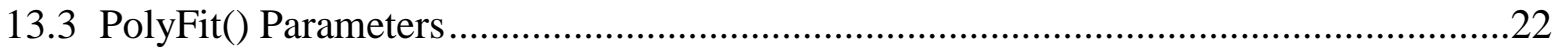

13.4 PolyFit() Simple Example ..............................................................................23

14. Example: Exponential Fits Using the PolyFit() Function 24 
15. Code Summary

16. References

Distribution List 


\section{List of Figures}

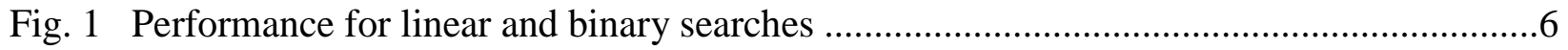

Fig. 2 A comparison of the Search() and PeriodicSearch() functions ....................................8

Fig. $3 y(x)$ represented by a finite number of points, with nearest-neighbor interpolation

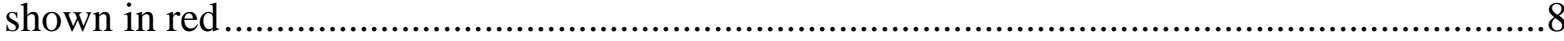

Fig. $4 y(x)$ represented by a finite number of points, with linear interpolation shown in red......10

Fig. $5 y(x)$ represented by a finite number of points, with cubic interpolation shown in red ......13

Fig. 6 Comparison of the NNInterp(), LinInterp(), and CubeInterp() functions ......................17

Fig. 7 NNInterp(), LinInterp(), and CubeInterp() functions used to create periodic plots ..........18

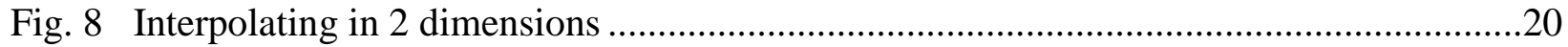

Fig. 9 2-dimensional interpolations using NNInterp(), LinInterp(), and CubeInterp() .............21

Fig. 10 Best-fit line calculated by the PolyFit() function ...................................................25 


\section{Acknowledgments}

I would like to thank Tim Fargus of the US Army Armament Research, Development, and Engineering Center's System Analysis Division. Tim provided technical and editorial recommendations that improved the quality of this report.

I would also like to thank Dr Douglas Chapman, Professor of Chemistry at Southern Oregon University, for the concepts he taught me in his "Computer Applications in Chemistry" class. Dr Chapman taught me that it's possible to use very simple computer code to solve complex problems. At the time, I didn't realize how valuable that lesson was. 
INTENTIONALLY LEFT BLANK. 


\section{Introduction}

This report documents a set of functions, written in $\mathrm{C}++$, that can be used to perform interpolations (nearest-neighbor, linear, and cubic) and to find coefficients for best-fit equations. Functions for working with periodic equations are included.

Measurements can be taken and stored using a variety of schemes. The interpolations described in this report are designed to work with data sets where measured values for all independent and dependent variables are explicitly stored. Furthermore, for data that has more than 1 independent variable, the measurements must all lie on some type of rectangular grid, where all grid locations are populated.

When measurements do not lie on some type of rectangular grid, interpolations become more difficult. For those types of data sets, KD-trees can be used to perform efficient nearest-neighbor searches, which can act as interpolations. The yKDTree namespace ${ }^{1}$ can be used to work with KD-trees using $\mathrm{C}++$.

The yBilinear namespace ${ }^{2}$ can be used for the special case of data sets with 2 independent variables, where all measurements lie on a rectangular grid, all grid locations are populated, and all grid lines are evenly spaced.

The functions that are described in this report have been grouped into the yInterp namespace, which is summarized at the end of this report. The yInterp namespace relies exclusively on standard $\mathrm{C}++$ operations and functions. However, example code that is included in this report makes use of the yRandom namespace ${ }^{3}$ for generating pseudorandom numbers, the yBmp namespace ${ }^{4}$ for creating images, and the yIo $2^{5}$ namespace for reading and parsing text files.

\section{Background}

My motivation for this project began with a task that involved interpolating a look-up table with 8 independent variables, some of which were periodic. Prior to this project, I had always written interpolators on an ad hoc basis. However, the thought of having to deal with an array that requires 8 indices to access a particular element (something like $A[\mathrm{i}][\mathrm{j}][\mathrm{k}][1][\mathrm{m}][\mathrm{n}][\mathrm{o}][\mathrm{p}]$ ) convinced me that I needed a more formal set of tools.

The task required that all code be written in $\mathrm{C}++$. To maximize portability, the code had to be written without the use of any platform-specific or $\mathrm{C}++11$ tools. Performance was a priority. In addition, I wanted a versatile set of tools that I could use for future projects. The tools needed to 
be simple to use and well-tested so that I could feel comfortable sharing them with other programmers.

\section{Searching Sorted Arrays: The BinarySearch() Function}

The BinarySearch() function uses a binary search algorithm to search arrays that are sorted into ascending order. For arrays that do not contain duplicate entries, the BinarySearch() function can be used to find a pointer $\mathbf{c}$ such that

$$
* \mathbf{c} \leq \mathbf{k}<*(\mathbf{c}+1)
$$

where $\mathbf{k}$ is key on which the search is based.

For arrays that contain duplicate entries, Eq. 1 needs to be slightly modified:

$$
* \mathbf{c} \leq \mathbf{k} \leq *(\mathbf{c}+1)
$$

Thus, if $\mathbf{k}$ is equal to an element that has one or more duplicates, $\mathbf{c}$ may point to any one of the duplicates.

Performance for the BinarySearch() function is $O(\log (n))$, which is demonstrated in Section 3.7.

\subsection{BinarySearch() Code}

template $<$ class T $>T^{*}$ BinarySearch $(/ /<======$ FIND THE POINTER $\mathrm{c} \mid * \mathrm{c}<=\mathrm{k}<*(\mathrm{c}+1)$ $T^{*} a, T^{*} b, / /<--A R R A Y$ START \& END POINTS (ARRAY MUST BE SORTED IN INC. ORDER)

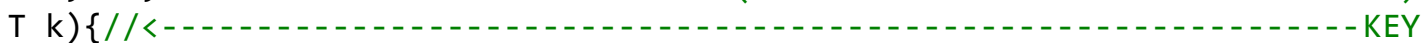
if $(\mathrm{k}<\mathrm{*} a)$ return $\mathrm{a}-1 ; / / \ldots \ldots \ldots$....... note that a-1 may point to an invalid address for $\left(T^{*} c ; k<*_{-}-b ; k>*^{*}\right.$ ? $\left.a=c: b=c+1\right) c=a+(b-a) / 2 ; / *_{-}>* /$ return $b$;

\}// YAGENAUT@GMAIL.COM LAST UPDATED 21JUL2014

\subsection{BinarySearch() Template Class}

T $\quad$ T must be a sortable data type.

\subsection{BinarySearch() Parameters}

a

b

$\mathbf{k}$ a points to the beginning of the array that will be searched. The array should be sorted into ascending order.

b points to one element past the end of the array that will be searched. Thus, $\mathbf{b}$ is used to define the end of the search region but is not included in the search. $\mathbf{b}$ should be greater than $\mathbf{a}$.

$\mathbf{k}$ is the key on which the search is based. 


\subsection{BinarySearch() Return Value}

For arrays that do not contain duplicate entries, the BinarySearch() function returns a pointer to the greatest array element that is less than or equal to $\mathbf{k}$. If $\mathbf{k}$ is less than the least array element, then $\mathbf{a}-1$ is returned. Care needs to be taken when accessing the return pointer, since $\mathbf{a}-1$ may not point to a valid address.

\subsection{BinarySearch() Simple Example}

The following example uses the BinarySearch() function to search a small sorted array that contains duplicates.

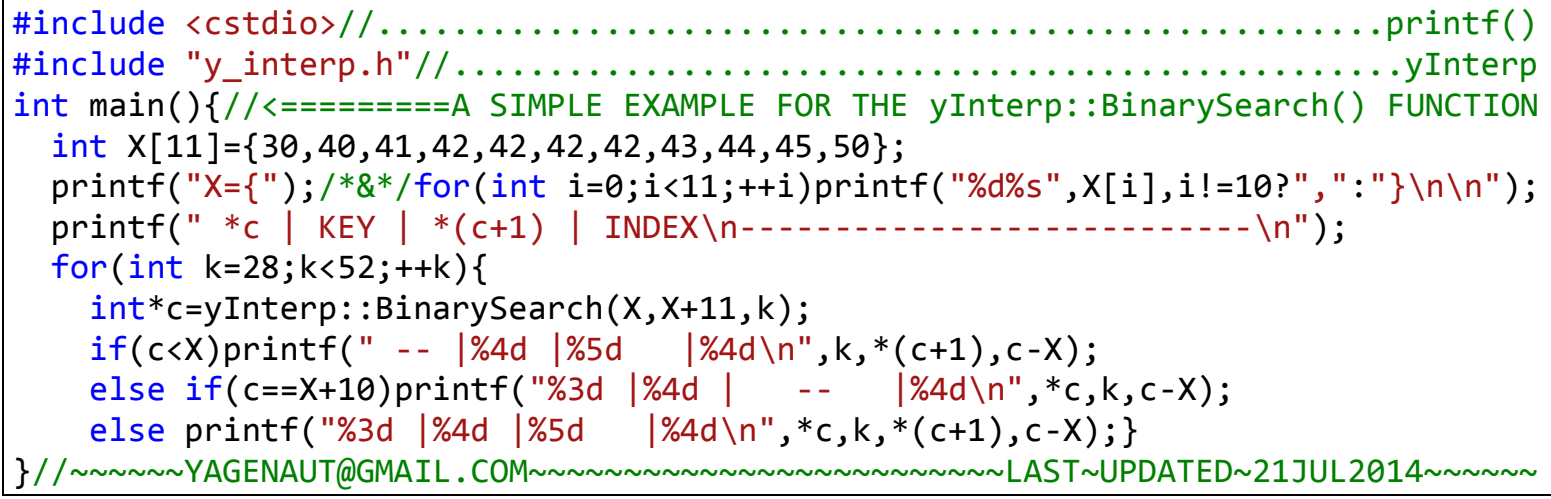

OUTPUT:

\begin{tabular}{|c|c|c|c|}
\hline \multicolumn{2}{|c|}{$\mathrm{X}=\{30,40,41,42,42,42,42,43,44,45,50\}$} \\
$* c$ & KEY & $*(c+1)$ & INDEX \\
\hline-- & 28 & 30 & -1 \\
-- & 29 & 30 & -1 \\
30 & 30 & 40 & 0 \\
30 & 31 & 40 & 0 \\
30 & 32 & 40 & 0 \\
30 & 33 & 40 & 0 \\
30 & 34 & 40 & 0 \\
30 & 35 & 40 & 0 \\
30 & 36 & 40 & 0 \\
30 & 37 & 40 & 0 \\
30 & 38 & 40 & 0 \\
30 & 39 & 40 & 0 \\
40 & 40 & 41 & 1 \\
41 & 41 & 42 & 2 \\
42 & 42 & 42 & 5 \\
43 & 43 & 44 & 7 \\
44 & 44 & 45 & 8 \\
45 & 45 & 50 & 9 \\
45 & 46 & 50 & 9 \\
45 & 47 & 50 & 9 \\
45 & 48 & 50 & 9 \\
45 & 49 & 50 & 9 \\
50 & 50 & -- & 10 \\
50 & 51 & -- & 10 \\
\hline & & &
\end{tabular}




\subsection{BinarySearch() Text Example}

The following example uses the BinarySearch() function to determine where a word should be inserted into a sorted list.

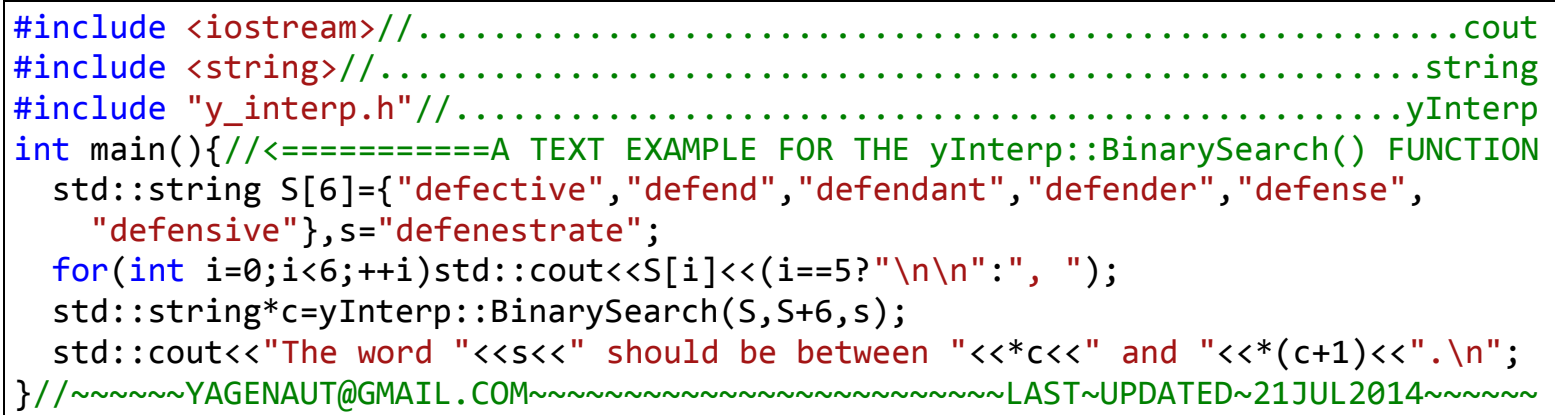

OUTPUT:

defective, defend, defendant, defender, defense, defensive

The word defenestrate should be between defender and defense.

\subsection{BinarySearch() Performance}

The following example begins by using the yRandom namespace to populate an array with $2^{14}$ pseudorandom numbers using the Mersenne twister 19937 algorithm. The array is then sorted using the sort() function. Average search times are calculated for searches performed using a linear search algorithm and the BinarySearch() function. Figure 1 presents a graph of the results.

The average search time for the LinearSearch() function is $O(n)$. The average search time for the BinarySearch() function is $O(\log (n))$. 


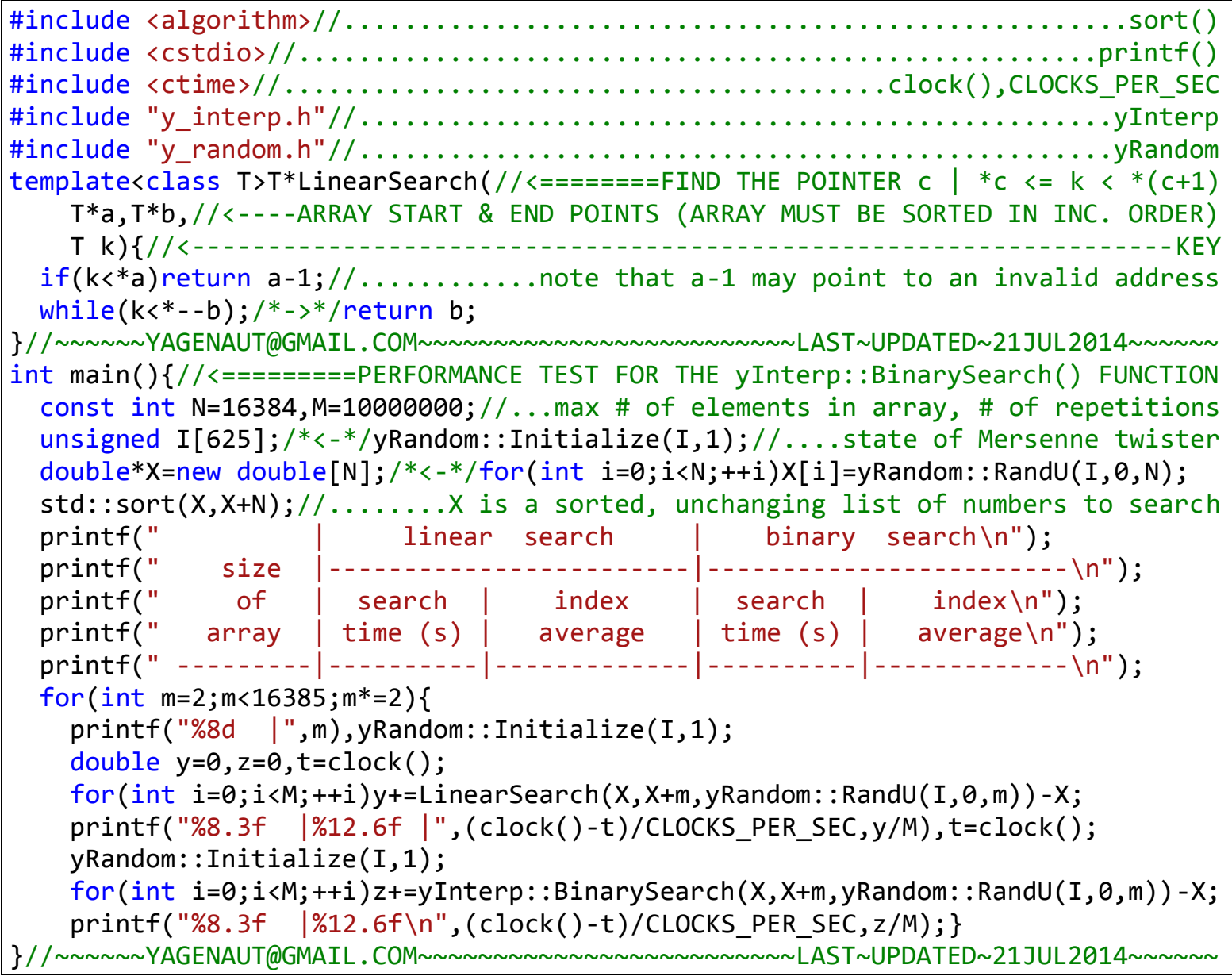
const int $\mathrm{N}=16384, \mathrm{M}=10000000 ; / / \ldots \max \#$ of elements in array, \# of repetitions unsigned I[625];/*<-*/yRandom: : Initialize(I,1);//... state of Mersenne twister double*X=new double $[N] ; / *<-* /$ for (int $i=0 ; i<N ;++i) X[i]=y \operatorname{Random:~:RandU}(I, 0, N)$; std:: $\operatorname{sort}(\mathrm{X}, \mathrm{X}+\mathrm{N}) ; / / \ldots \ldots \ldots \mathrm{X}$ is a sorted, unchanging list of numbers to search printf(" linear search | binary search $\backslash n ")$;

OUTPUT:

\begin{tabular}{|c|c|c|c|c|}
\hline & linear & search & binary & search \\
\hline size & & & - - - - & - - - - - - - - - - \\
\hline of & search & index & search & index \\
\hline array & time $(s)$ & average & time (s) & average \\
\hline & - - - - - - - & & & - \\
\hline 2 & 0.140 & -0.731079 & 0.125 & -0.731079 \\
\hline 4 & 0.140 & 0.555495 & 0.156 & 0.555495 \\
\hline 8 & 0.203 & 1.833760 & 0.224 & 1.833760 \\
\hline 16 & 0.244 & 5.133788 & 0.234 & 5.133788 \\
\hline 32 & 0.312 & 13.015384 & 0.327 & 13.015384 \\
\hline 64 & 0.390 & 31.287311 & 0.359 & 31.287311 \\
\hline 128 & 0.531 & 66.159459 & 0.421 & 66.159459 \\
\hline 256 & 0.936 & 128.260683 & 0.530 & 128.260683 \\
\hline 512 & 1.545 & 258.242078 & 0.592 & 258.242078 \\
\hline 1024 & 2.902 & 519.408822 & 0.671 & 519.408822 \\
\hline 2048 & 5.678 & 1035.587832 & 0.733 & 1035.587832 \\
\hline 4096 & 11.217 & 2054.054591 & 0.795 & 2054.054591 \\
\hline 8192 & 22.142 & 4091.340401 & 0.873 & 4091.340401 \\
\hline 16384 & 43.945 & 8193.415153 & 0.968 & 8193.415153 \\
\hline
\end{tabular}




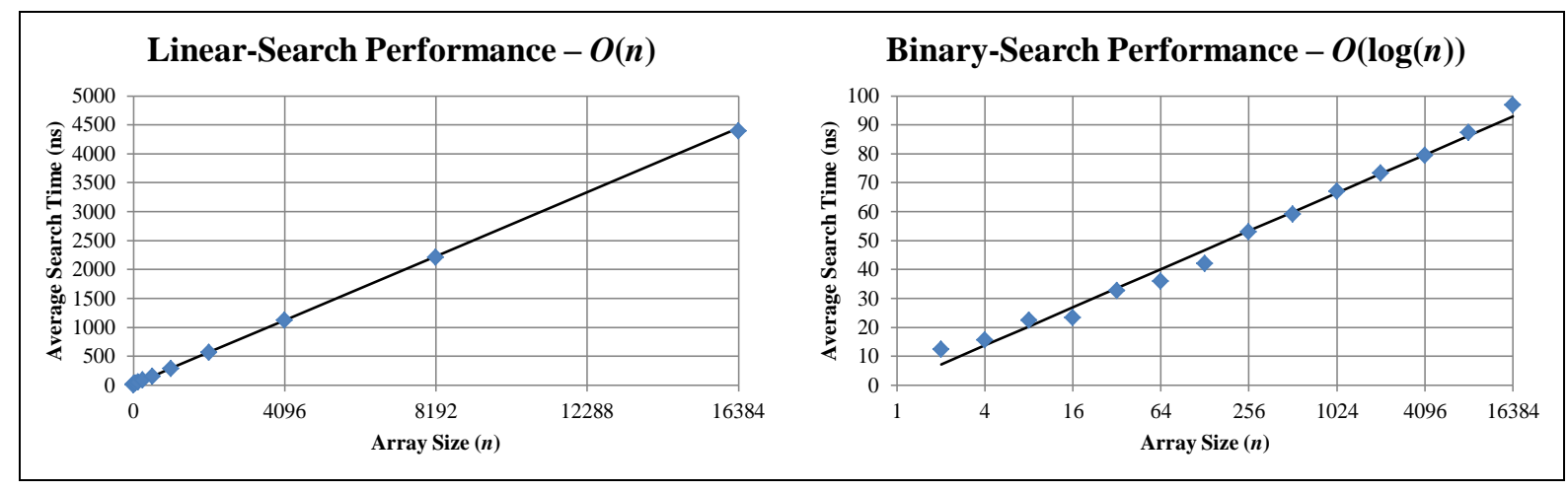

Fig. 1 Performance for linear and binary searches (right graph is semi-log)

\section{Searching Sorted Arrays: The PeriodicSearch() Function}

The PeriodicSearch() function uses the BinarySearch() function to search arrays that are associated with periodic functions, such as $f$, where

$$
f(x+n p)=f(x) .
$$

Thus, the PeriodicSearch() function can be used to find a pointer $\mathbf{c}$ such that

$$
* \mathbf{c} \leq \mathbf{k}+n \mathbf{p}<*(\mathbf{c}+1),
$$

where $\mathbf{k}$ and $\mathbf{p}$ are user supplied values and the integer $n$ is chosen such that

$$
* \mathbf{a} \leq \mathbf{k}+n \mathbf{p}<* \mathbf{a}+\mathbf{p} .
$$

\subsection{PeriodicSearch() Code}

template<class T>T*PeriodicSearch(//<=======FOR ARRAYS WITH PERIODIC VARIABLES $T^{*} \mathrm{a}, \mathrm{T}^{*} \mathrm{~b}, / /<--S T A R T I N G$ \& ENDING POINTS (ARRAY MUST BE SORTED IN INC. ORDER)

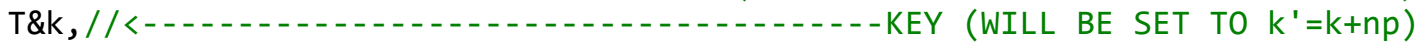

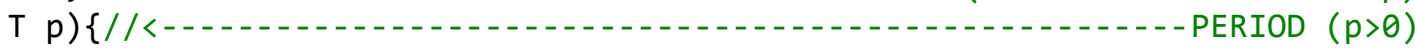
return BinarySearch $(a, b, k=f \bmod (k-* a, p)+* a+(k-* a<0 ? p: 0))$;

\}// YAGENAUT@GMAIL.COM LAST UPDATED 21JUL 2014

\subsection{PeriodicSearch() Template Class}

$\mathbf{T}$

T must be a sortable data type.

\subsection{PeriodicSearch() Parameters}

$\mathbf{a}$

a points to the beginning of the array that will be searched. The array should be sorted into ascending order. 
b b points to one element past the end of the array that will be searched. Thus, $\mathbf{b}$ is used to define the end of the search region but is not included in the search. $\mathbf{b}$ should be greater than a.

$\mathbf{k} \quad \mathbf{k}$ is the key on which the search is based. Note that before the function returns, $\mathbf{k}$ is set to $k^{\prime}$, where $k^{\prime}=\mathbf{k}+n \mathbf{p}$.

$\mathbf{p} \quad \mathbf{p}$ is the period of the function associated with the array. Values for $\mathbf{p}$ are typically chosen such that $\mathbf{p}>*(\mathbf{b}-1)-* \mathbf{a}$. In no case should $\mathbf{p}$ be equal to zero.

\subsection{PeriodicSearch() Return Value}

The PeriodicSearch() function returns a pointer that satisfies the requirements for $\mathbf{c}$ in Eq. 4 .

\subsection{PeriodicSearch() Example}

The following example begins by creating a pair of arrays that are used to store independent and dependent variables that approximate Eq. 6.

$$
y(x)=\sin (2 \pi x / 5)+1
$$

The BinarySearch() and PeriodicSearch() functions are then used to retrieve dependent variables based on a selection of independent variables. Figure 2 presents a pair of graphs that display the results of the searches.

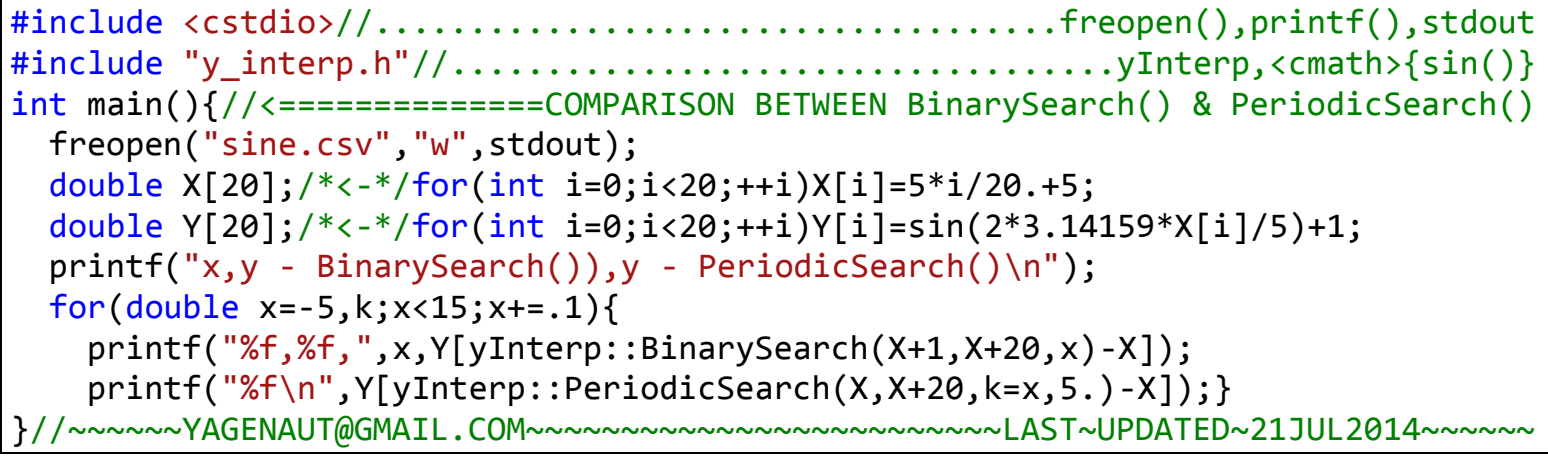




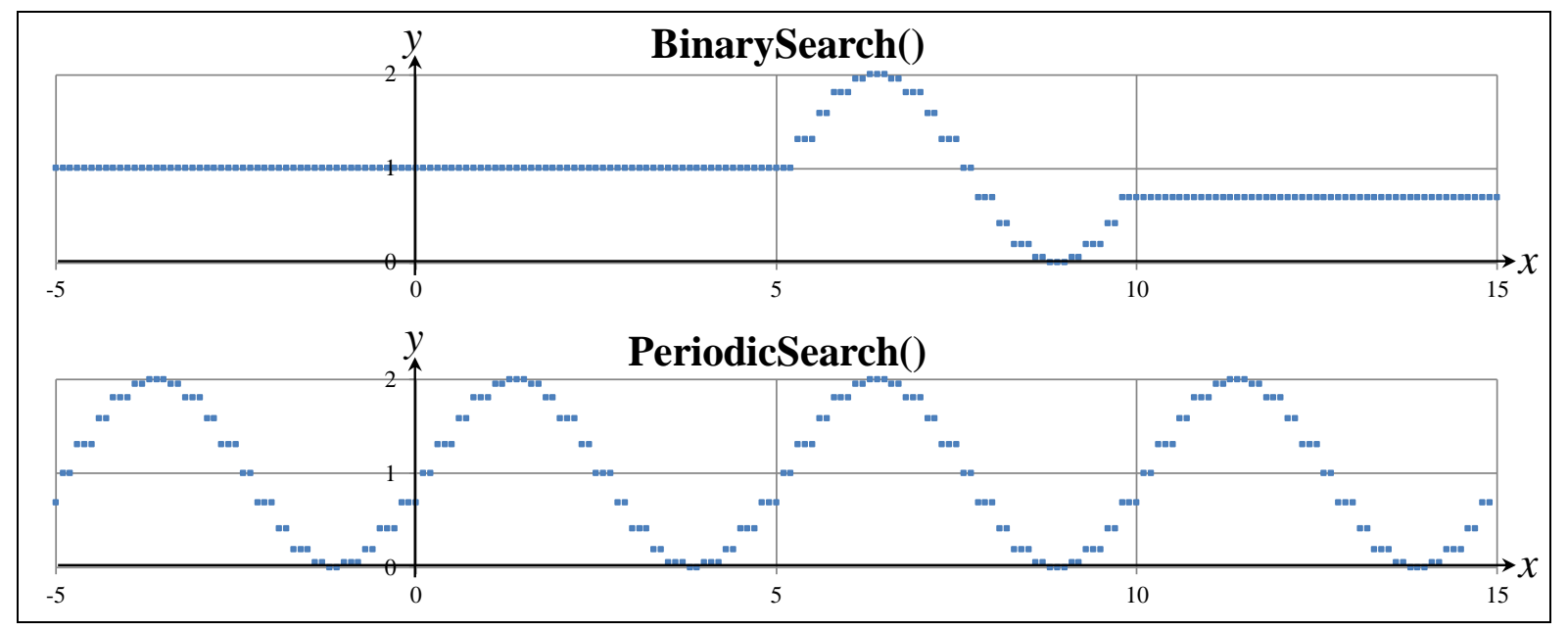

Fig. 2 A comparison of the Search() and PeriodicSearch() functions

\section{Performing Nearest-Neighbor Interpolations: The NNInterp() Function}

Suppose that some function $y(x)$ is represented by a finite set of points $\left(x_{i}, y_{i}\right)$, such as is shown in Fig. 3.

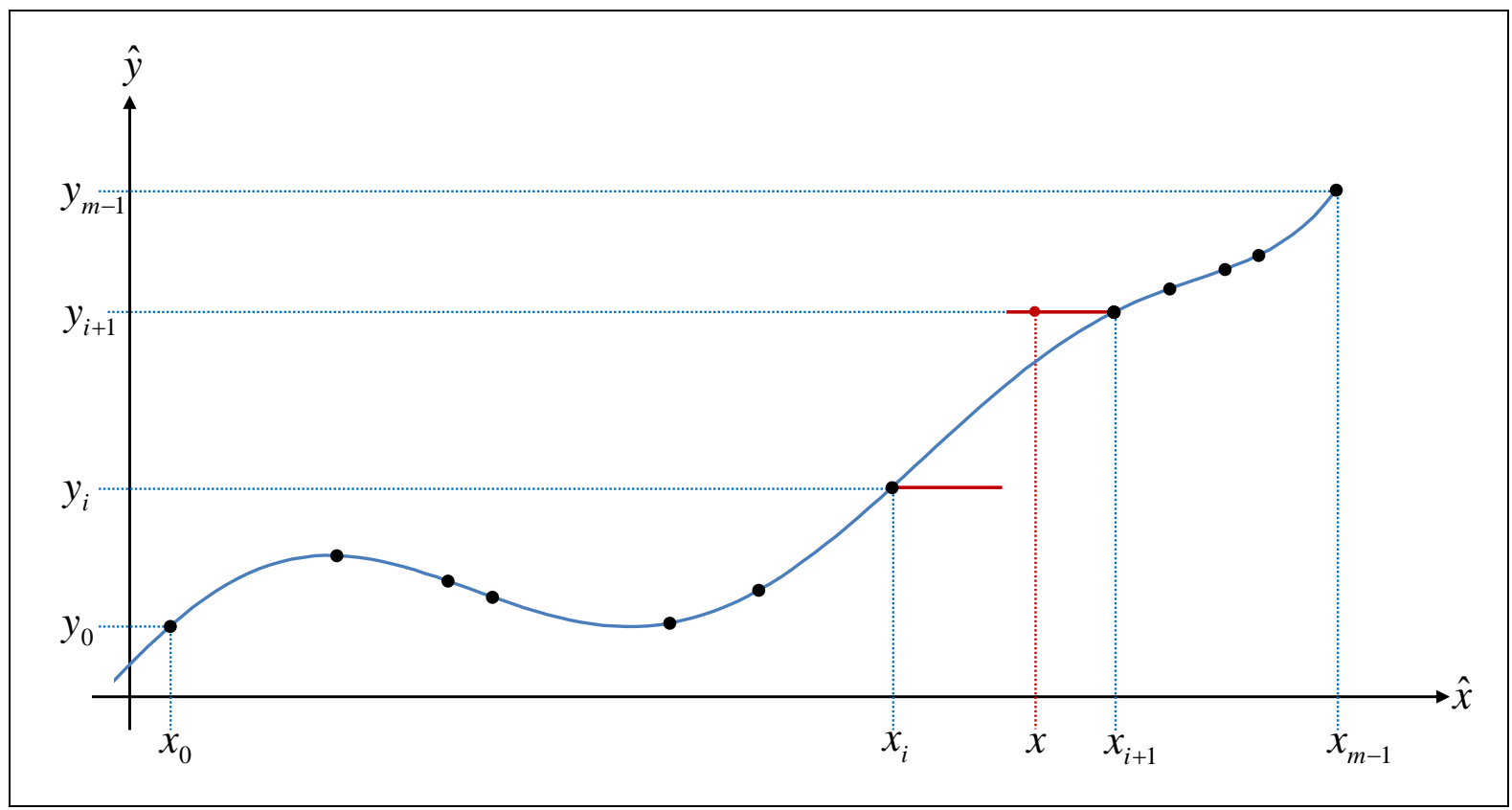

Fig. $3 y(x)$ represented by a finite number of points, with nearest-neighbor interpolation shown in red

The NNInterp() function uses Eq. 7, represented by the solid red line in Fig. 3, to approximate $y(x)$ : 


$$
y(x)=\left\{\begin{array}{ll}
y_{i+1} & \text { for } x>\frac{x_{i}+x_{i+1}}{2} \\
y_{i} & \text { otherwise }
\end{array} .\right.
$$

\subsection{NNInterp() Code}

template<class $T>T$ NNInterp $(/ /<=================N E A R E S T-N E I G H B O R$ INTERPOLATOR const $T^{*} \mathrm{X}, / /<-\cdots-\cdots$ BRACKETING $X$ VALUES (*X AND $X[1]$ MUST BE VALID)

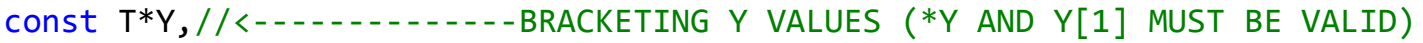
T $\mathrm{x})\{/ /<-\cdots-\cdots-\cdots$ - VALUE TO INTERPOLATE AT (TYPICALLY, $* \mathrm{X}<=\mathrm{x}<=\mathrm{x}[1]$ ) return $\mathrm{X}>(* \mathrm{X}+\mathrm{X}[1]) / 2$ ? $\mathrm{Y}[1]: * \mathrm{Y}$;

\}// YAGENAUT@GMAIL.COM LAST UPDATED 21JUL 2014

\subsection{NNInterp() Template Class}

$\mathbf{T} \quad \mathbf{T}$ is typically a floating-point data type.

\subsection{NNInterp() Parameters}

$\mathbf{X} \quad \mathbf{X}$ points to an array that is used to store values for $x_{i}$ from Eq. 7. Specifically, $* \mathbf{X}=x_{i}$ and $\mathbf{X}[1]=x_{i+1}$. Thus, both $\mathbf{X}$ and $\mathbf{X}+1$ must point to valid addresses.

$\mathbf{Y} \quad \mathbf{Y}$ points to an array that is used to store values for $y_{i}$ from Eq. 7. Specifically, $* \mathbf{Y}=y_{i}$ and $\mathbf{Y}[1]=y_{i+1}$. Thus, both $\mathbf{Y}$ and $\mathbf{Y}+1$ must point to valid addresses.

$\mathbf{x} \quad \mathbf{x}$ represents the independent variable $x$ from Eq. 7 .

\subsection{NNInterp() Return Value}

The NNInterp() function returns $y$ from Eq. 7 .

\subsection{NNInterp() Simple Example}

The following example begins by creating a pair of arrays that are used to store independent and dependent variables that approximate Eq. 6. Next, the BinarySearch() function is used to find a pointer that conforms to Eq. 1, which is then converted to an index. Finally, the NNInterp() function is used to approximate $y(7.180)$.

Note that the value for the BinarySearch() parameter $\mathbf{a}$ is set to $\mathbf{X}+1$ to avoid the possibility of accessing the $\mathbf{X}-1$ element. Similarly, the value for the BinarySearch() parameter $\mathbf{b}$ is set to $\mathbf{X}+19$ to avoid the possibility of accessing the $\mathbf{X}+20$ element. 


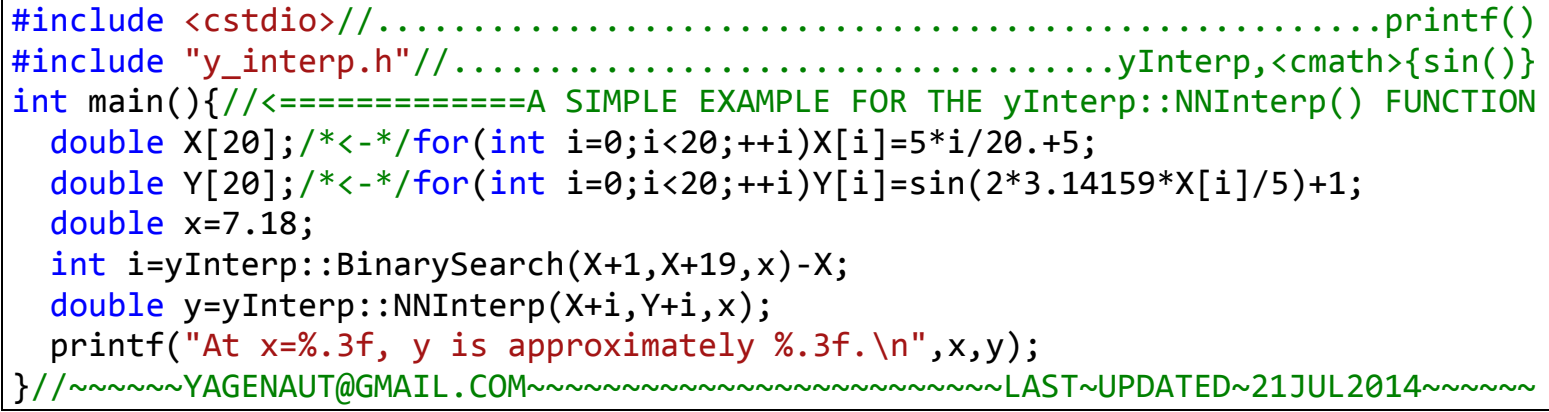

OUTPUT:

At $\mathrm{x}=7.180, \mathrm{y}$ is approximately 1.309 .

\section{Performing Linear Interpolations: The LinInterp() Function}

Suppose that some function $y(x)$ is represented by a finite set of points $\left(x_{i}, y_{i}\right)$, such as is shown in Fig. 4.

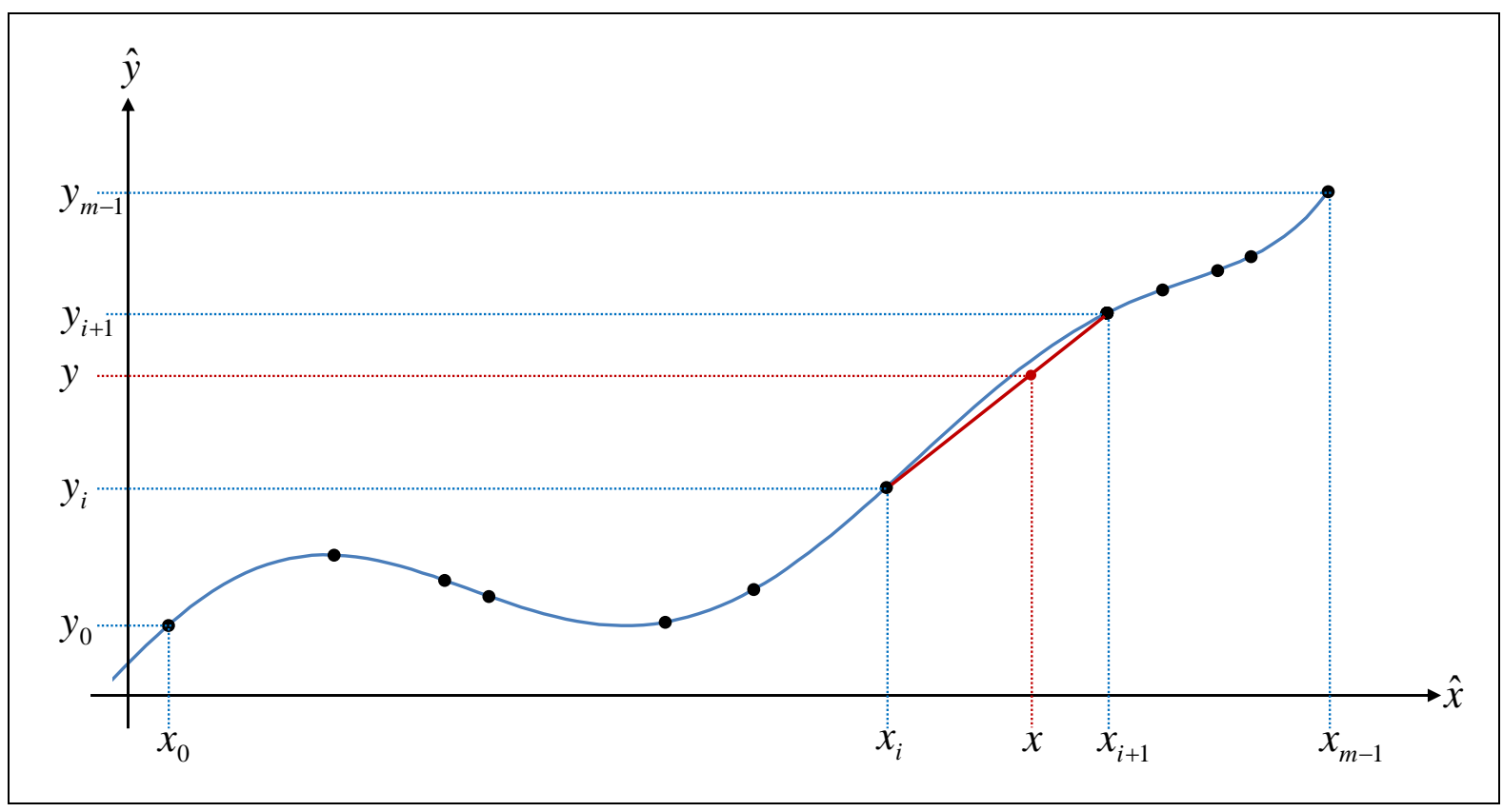

Fig. $4 y(x)$ represented by a finite number of points, with linear interpolation shown in red

The LinInterp() function uses Eq. 8, represented by the solid red line in Fig. 4, to approximate $y(x)$ : 


$$
y(x)=y_{i}+\left(y_{i+1}-y_{i}\right) \frac{x-x_{i}}{x_{i+1}-x_{i}} .
$$

\subsection{LinInterp() Code}

template<class $\mathrm{T}>\mathrm{T}$ LinInterp $(/ /<===========================\mathrm{LINEAR}$ INTERPOLATOR const $T^{*} X, / /<-\cdots-\cdots-\cdots$ BRACKETING $X$ VALUES (*X AND $X[1]$ MUST BE VALID) const $\mathrm{T}^{*} \mathrm{Y}, / / / \cdots^{-\cdots}-\cdots-\cdots$ BRACKETING $\mathrm{Y}$ VALUES (*Y AND $\mathrm{Y}[1]$ MUST BE VALID) T $\mathrm{x})\left\{/ /<-\cdots-\cdots-\cdots\right.$ VALUE TO INTERPOLATE AT (TYPICALLY, ${ }^{*} \mathrm{X}<=\mathrm{x}<=\mathrm{x}[1]$ ) return $* \mathrm{Y}+(\mathrm{Y}[1]-* \mathrm{Y}) *(\mathrm{X}-* \mathrm{X}) /(\mathrm{X}[1]-* \mathrm{X})$;

\}// YAGENAUT@GMAIL.COM LAST UPDATED 21JUL2014

\subsection{LinInterp() Template Class}

$\mathbf{T} \quad \mathbf{T}$ is typically a floating-point data type.

\subsection{LinInterp() Parameters}

$\mathbf{X} \mathbf{X}$ points to an array that is used to store values for $x_{i}$ from Eq. 8. Specifically, $* \mathbf{X}=x_{i}$ and $\mathbf{X}[1]=x_{i+1}$. Thus, both $\mathbf{X}$ and $\mathbf{X}+1$ must point to valid addresses.

$\mathbf{Y} \quad \mathbf{Y}$ points to an array that is used to store values for $y_{i}$ from Eq. 8. Specifically, $* \mathbf{Y}=y_{i}$ and $\mathbf{Y}[1]=y_{i+1}$. Thus, both $\mathbf{Y}$ and $\mathbf{Y}+1$ must point to valid addresses.

$\mathbf{x} \quad \mathbf{x}$ represents the independent variable $x$ from Eq. 8.

\subsection{LinInterp() Return Value}

The LinInterp() function returns $y$ from Eq. 8 .

\subsection{LinInterp() Simple Example}

The following example begins by creating a pair of arrays that are used to store independent and dependent variables that approximate Eq. 6. Next, the BinarySearch() function is used to find a pointer that conforms to Eq. 1, which it then converted to an index. Finally, the LinInterp() function is used to approximate $y(7.180)$.

Note that the value for the BinarySearch() parameter $\mathbf{a}$ is set to $\mathbf{X}+1$ to avoid the possibility of accessing the $\mathbf{X}-1$ element. Similarly, the value for the BinarySearch() parameter $\mathbf{b}$ is set to $\mathbf{X}+19$ to avoid the possibility of accessing the $\mathbf{X}+20$ element. 


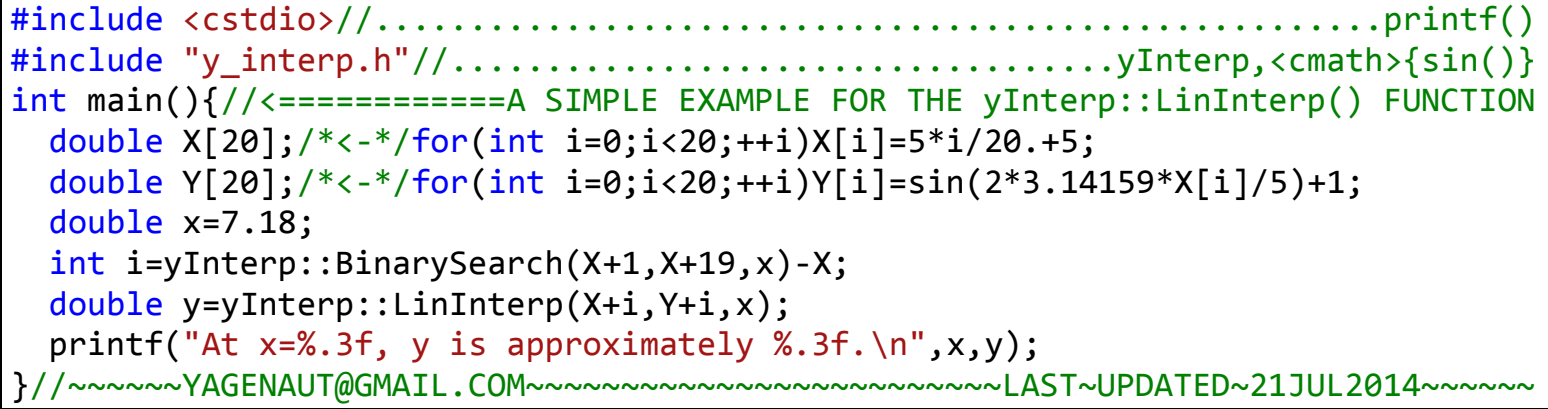

OUTPUT:

At $\mathrm{x}=7.180, \mathrm{y}$ is approximately 1.387 .

\section{Performing Cubic Interpolations: The CubeInterp() Function}

A cubic Hermite spline is a third-degree-polynomial interpolating function that is uniquely determined by 2 endpoint positions $\left(\vec{p}_{0}\right.$ and $\vec{p}_{1}$ ) and tangent vectors at the 2 endpoint positions $\left(\vec{m}_{0}\right.$ and $\vec{m}_{1}$, respectively):

$$
\vec{p}(t)=H_{0}(t) \vec{p}_{0}+H_{1}(t) \vec{m}_{0}+H_{2}(t) \vec{p}_{1}+H_{3}(t) \vec{m}_{1}
$$

$H_{0}(t), H_{1}(t), H_{2}(t)$, and $H_{3}(t)$ are known as Hermite basis functions and are given by Eqs.

10-13. $t$ is the linearly scaled distance from $\vec{p}_{0}$ to $\vec{p}_{1}$ such that at $\vec{p}_{0}, t=0$ and at $\vec{p}_{1}, t=1$.

$$
\begin{gathered}
H_{0}(t) \equiv 2 t^{3}-3 t^{2}+1 . \\
H_{1}(t) \equiv t^{3}-2 t^{2}+t \\
H_{2}(t) \equiv-2 t^{3}+3 t^{2} \\
H_{3}(t) \equiv t^{3}-t^{2} .
\end{gathered}
$$

Suppose that some function $y(x)$ is represented by a finite set of points $\left(x_{i}, y_{i}\right)$, such as is shown in Fig. 5. 


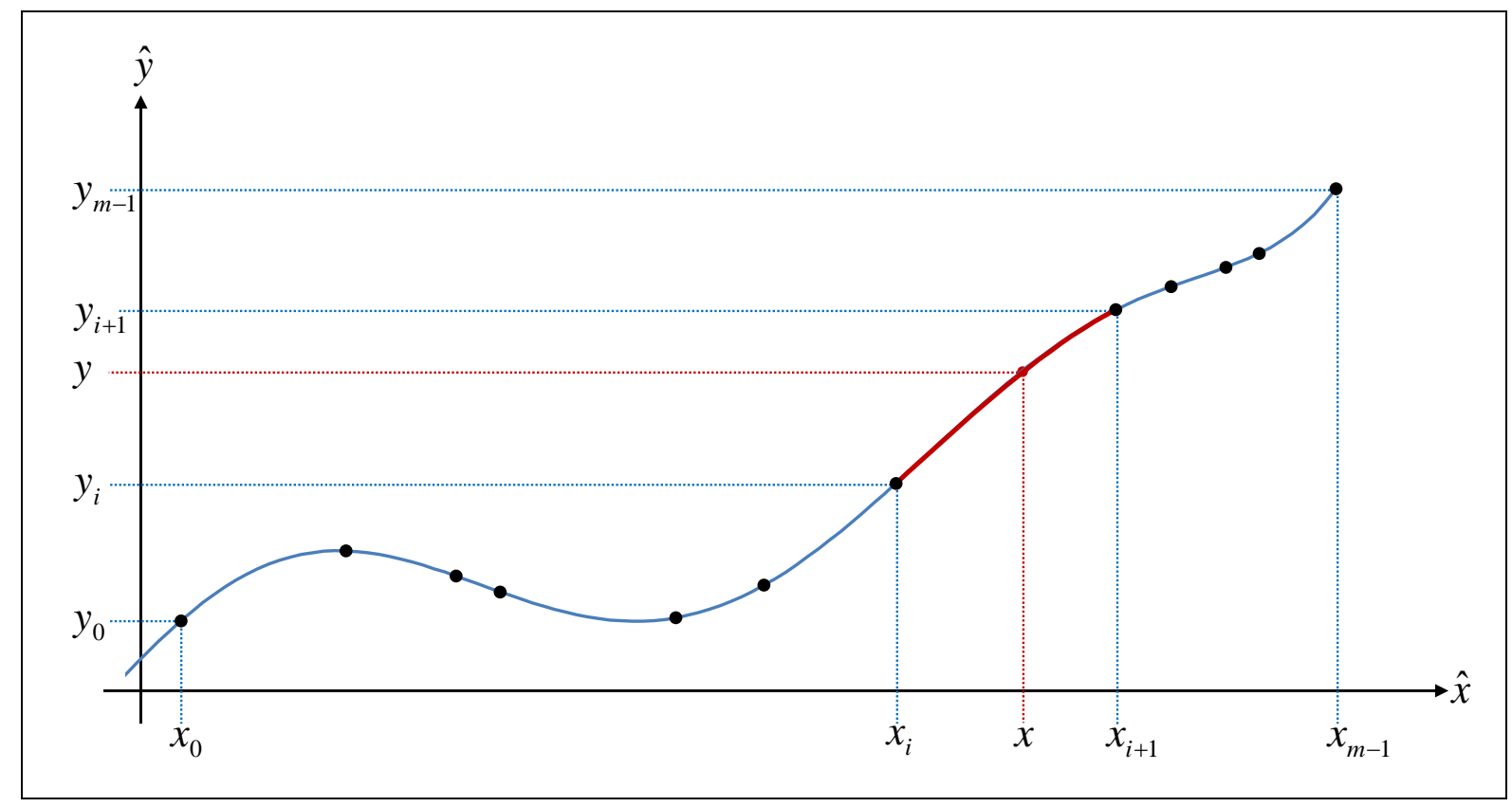

Fig. $5 y(x)$ represented by a finite number of points, with cubic interpolation shown in red

From Eq. 9 (and noting that $\left.H_{0}(t)=1-H_{2}(t)\right)$,

$$
y(t)=\left.H_{1}(t) \frac{d y}{d t}\right|_{t=0}+H_{2}(t)\left(y_{i+1}-y_{i}\right)+\left.H_{3}(t) \frac{d y}{d t}\right|_{t=1}+y_{i},
$$

Eq. 15 can be used to convert from the scaled independent variable $t$ to the general independent variable $x$.

$$
t=\frac{x-x_{i}}{x_{i+1}-x_{i}}
$$

Eq. 16, along with the chain rule, can be used to convert the derivatives.

$$
\frac{d t}{d x}=\frac{1}{x_{i+1}-x_{i}}
$$

Thus,

$$
\left.\frac{d y}{d t}\right|_{t=0}=\left(x_{i+1}-x_{i}\right) m_{i}
$$

and

$$
\left.\frac{d y}{d t}\right|_{t=1}=\left(x_{i+1}-x_{i}\right) m_{i+1} \text {, }
$$


where

$$
\left.m_{i} \equiv \frac{d y}{d x}\right|_{x=x_{i}}
$$

Substituting Eqs. 11-13, 17, and 18 into Eq. 14, then regrouping terms, results in the form of the cubic interpolating function that is used by the CubeInterp() function:

$$
y=y_{i}+\left(x-x_{i}\right)\left[\left\{\left(m_{i}+m_{i+1}\right) t-\left(2 m_{i}+m_{i+1}\right)\right\} t+m_{i}\right]+\left(y_{i+1}-y_{i}\right)(3-2 t) t^{2} .
$$

\subsection{CubeInterp () Code}

template $<$ class $T>T$ CubeInterp $(/ /<=========$ CUBIC (HERMITE SPLINE) INTERPOLATOR const $T^{*} \mathrm{X}, / /<-\cdots-\cdots$ const $\mathrm{T}^{*} \mathrm{Y}, / / /<-\cdots-\cdots$

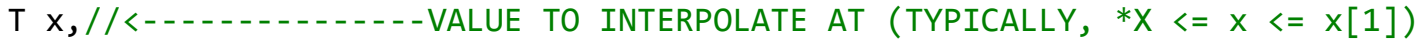
$\mathrm{T} \mathrm{m} 0, \mathrm{~T} \mathrm{~m} 1)\{/ /<\ldots \ldots \ldots$ SLOPES AT $* \mathrm{X}$ AND X[1] $\mathrm{T} \mathrm{t}=(\mathrm{x}-* \mathrm{X}) /(\mathrm{X}[1]-* \mathrm{X})$; return $* \mathrm{Y}+(\mathrm{X}-* \mathrm{X}) *(((\mathrm{~m} \theta+\mathrm{m} 1) * \mathrm{t}-(2 * \mathrm{~m} \theta+\mathrm{m} 1)) * \mathrm{t}+\mathrm{m} \theta)+(\mathrm{Y}[1]-* \mathrm{Y}) *(3-2 * \mathrm{t}) * \mathrm{t} * \mathrm{t}$; \}// YAGENAUT@GMAIL.COM LAST UPDATED 21JUL 2014

\subsection{CubeInterp() Template Class}

$\mathbf{T}$

$\mathbf{T}$ is typically a floating-point data type.

\subsection{CubeInterp() Parameters}

$\mathbf{X} \mathbf{X}$ points to an array that is used to store values for $x_{i}$ from Eq. 20. Specifically, $* \mathbf{X}=x_{i}$ and $\mathbf{X}[1]=x_{i+1}$. Thus, both $\mathbf{X}$ and $\mathbf{X}+1$ must point to valid addresses.

$\mathbf{Y} \quad \mathbf{Y}$ points to an array that is used to store values for $y_{i}$ from Eq. 20. Specifically, $* \mathbf{Y}=y_{i}$ and $\mathbf{Y}[1]=y_{i+1}$. Thus, both $\mathbf{Y}$ and $\mathbf{Y}+1$ must point to valid addresses.

m0 $\mathbf{m 0}$ represents $m_{i}$, the slope of the interpolating function at $x_{i}$.

$\mathbf{m} 1 \mathbf{m} 1$ represents $m_{i+1}$, the slope of the interpolating function at $x_{i+1}$.

$\mathbf{x} \quad \mathbf{x}$ represents the independent variable $x$ from Eq. 20 .

\subsection{CubeInterp() Return Value}

The CubeInterp() function returns $y$ from Eq. 20 .

\subsection{CubeInterp() Simple Example}

The following example begins by creating a pair of arrays that are used to store independent and dependent variables that approximate Eq. 6. Next, the BinarySearch() function is used to find a pointer that conforms to Eq. 1, which is then converted to an index. Finally, the CubeInterp() 
function is used to approximate $y(7.180)$. For simplicity, $m_{i}$ and $m_{i+1}$ have been set to zero. Section 8 presents a function that can be used to find values for $m_{i}$ and $m_{i+1}$.

Note that the value for the BinarySearch() parameter a is set to $\mathbf{X}+1$ to avoid the possibility of accessing the $\mathbf{X}$-1 element. Similarly, the value for the BinarySearch() parameter $\mathbf{b}$ is set to $\mathbf{X}+19$ to avoid the possibility of accessing the $\mathbf{X}+20$ element.

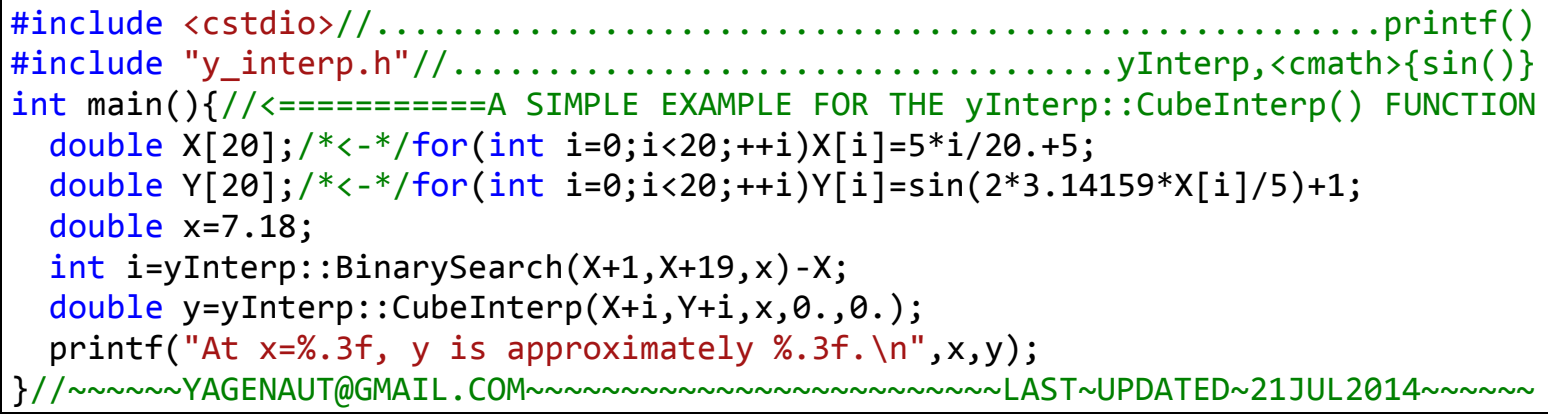

OUTPUT:

At $\mathrm{x}=7.180, \mathrm{y}$ is approximately 1.362 .

\section{Cardinal Splines: The CardinalSlope() Function}

The CardinalSlope() function uses Eq. 21 to calculate the CubeInterp()-function input parameters m0 and $\mathbf{m 1}$.

$$
m_{i}=(1-t) \frac{y_{i+1}-y_{i-1}}{x_{i+1}-x_{i-1}}
$$

$t$ is sometimes referred to as a tension parameter and is typically limited to the interval $[0,1]$.

\subsection{CardinalSlope() Code}

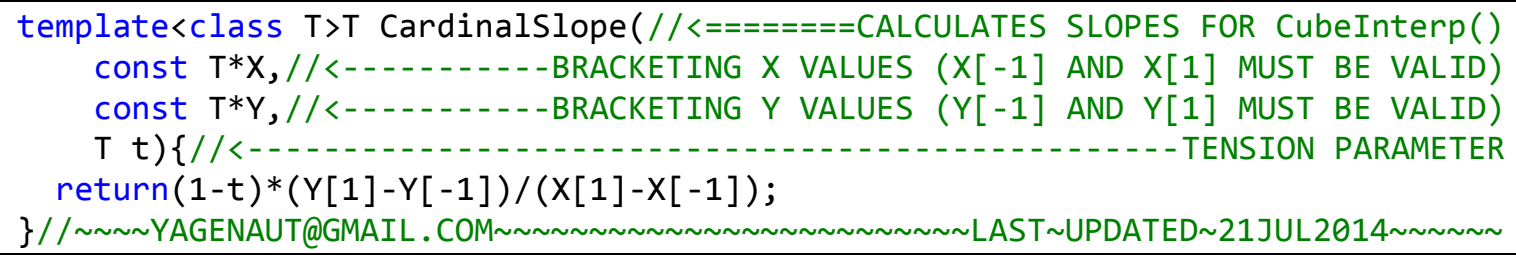

\subsection{CardinalSlope() Template Class}

$\mathbf{T}$ is typically a floating-point data type. 


\subsection{CardinalSlope() Parameters}

$\mathbf{X} \quad \mathbf{X}$ points to an array that is used to store values for $x_{i}$ from Eq. 21. Specifically, $\mathbf{X}[-1]=x_{i-1}$ and $\mathbf{X}[1]=x_{i+1}$. Thus, both $\mathbf{X}-1$ and $\mathbf{X}+1$ must point to valid addresses.

Y $\quad \mathbf{Y}$ points to an array that is used to store values for $y_{i}$ from Eq. 21. Specifically, $\mathbf{Y}[-1]=y_{i-1}$ and $\mathbf{Y}[1]=y_{i+1}$. Thus, both $\mathbf{Y}-1$ and $\mathbf{Y}+1$ must point to valid addresses.

$\mathbf{t} \quad \mathbf{t}$ represents $t$, the tension parameter from Eq. 21.

Because the CardinalSlope() function looks at points to either side of the bracketing points, extra care needs to be taken when selecting $\mathbf{X}$ and $\mathbf{Y}$.

\subsection{CardinalSlope() Return Value}

The CardinalSlope() function returns $m_{i}$ from Eq. 21 .

\subsection{CardinalSlope() Simple Example}

The following example begins by creating a pair of arrays that are used to store independent and dependent variables that approximate Eq. 6. Next, the BinarySearch() function is used to find a pointer that conforms to Eq. 1, which is then converted to an index. Finally, the CubeInterp() function is used to approximate $y(7.180)$.

Note that the value for the BinarySearch() parameter a has been set to $\mathbf{X}+1$ to avoid the possibility of accessing the $\mathbf{X}-1$ element. Similarly, the value for the BinarySearch() parameter $\mathbf{b}$ has been set to $\mathbf{X}+19$ to avoid the possibility of accessing the $\mathbf{X}+20$ element.

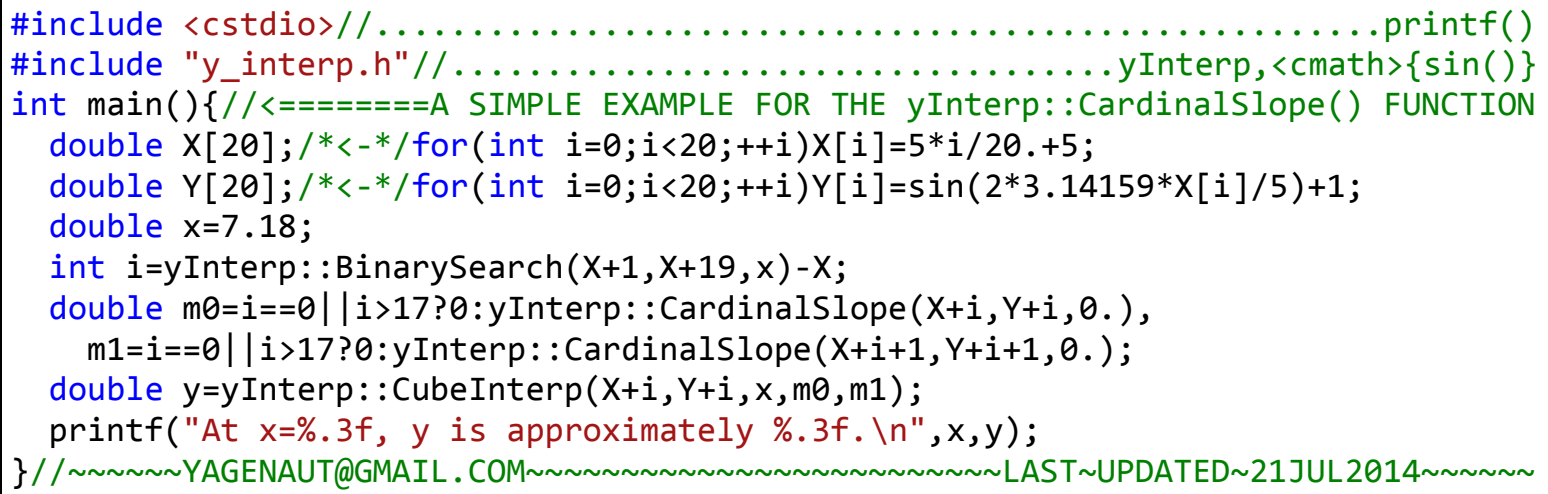

OUTPUT:

At $\mathrm{x}=7.180, \mathrm{y}$ is approximately 1.391 . 


\section{Example: Comparing Interpolating Functions}

The following example code creates a comma-separated text file that can be used to create the graph shown in Fig. 6. The plot relating to the CubeInterp() function relies on slopes that are calculated using the CardinalSlope() function (except for the endpoints, where the slopes are set to zero).
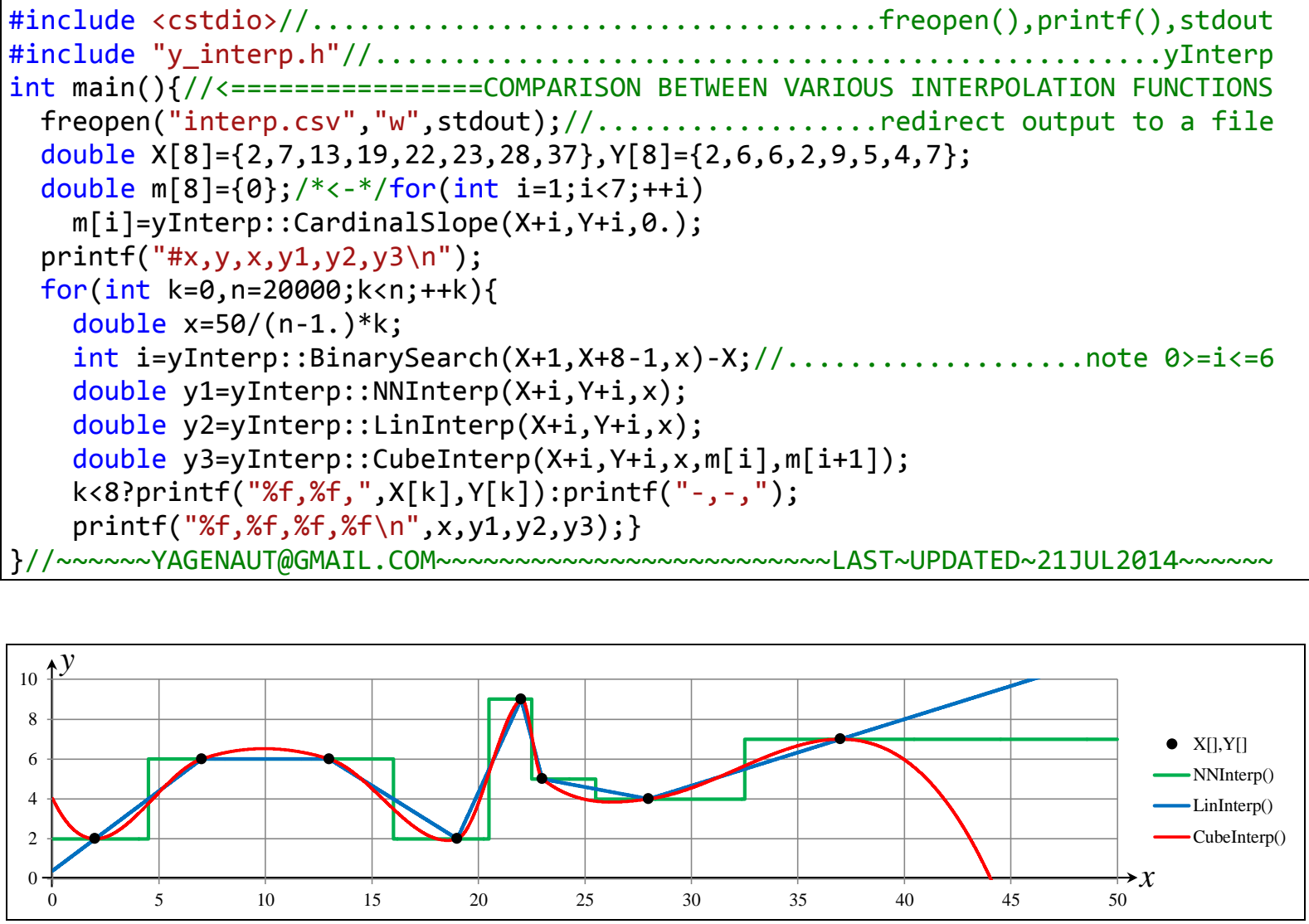

Fig. 6 Comparison of the NNInterp(), LinInterp(), and CubeInterp() functions

\section{Example: Performing Periodic Interpolations}

The following example code begins by setting up a set of points that are used to represent a periodic function with a period equal to 10.0. Next, the NNInterp(), LinInterp(), and CubInterp() functions are used to create values for a comma-separated text file. The graph shown in Fig. 7 represents the contents of the output file. 

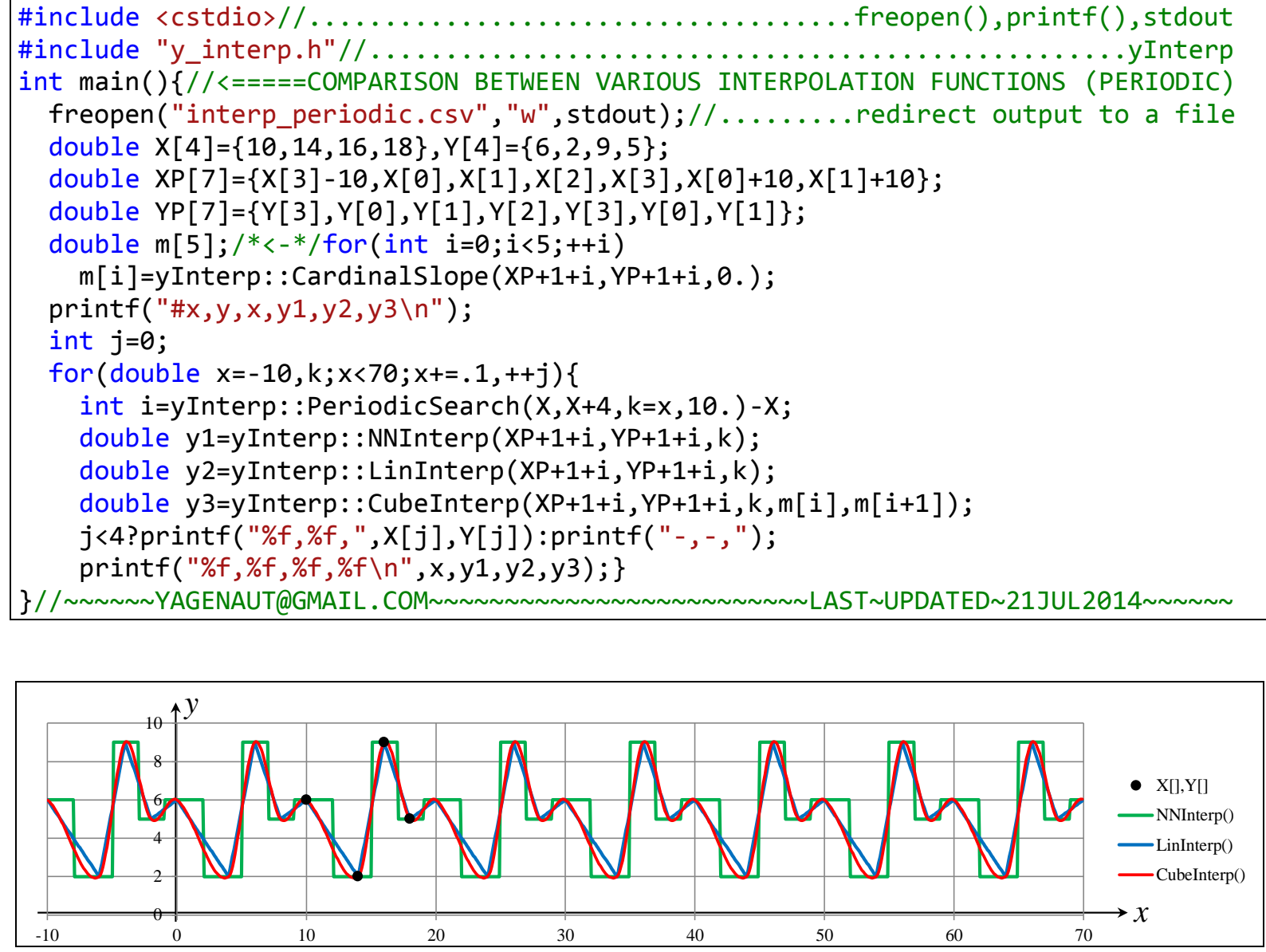

Fig. 7 NNInterp(), LinInterp(), and CubeInterp() functions used to create periodic plots

\section{Example: Determining Interpolation Performance}

The following example can be used to test the performance of the NNInterp(), LinInterp(), and CubeInterp() functions.

The output was generated by compiling the code using Microsoft's Visual Studio C++ 2010 Express compiler, with the output set to "release" mode. Surprisingly, for this scenario, the LinInterp() and CubeInterp() functions outperform the NNInterp() function. 


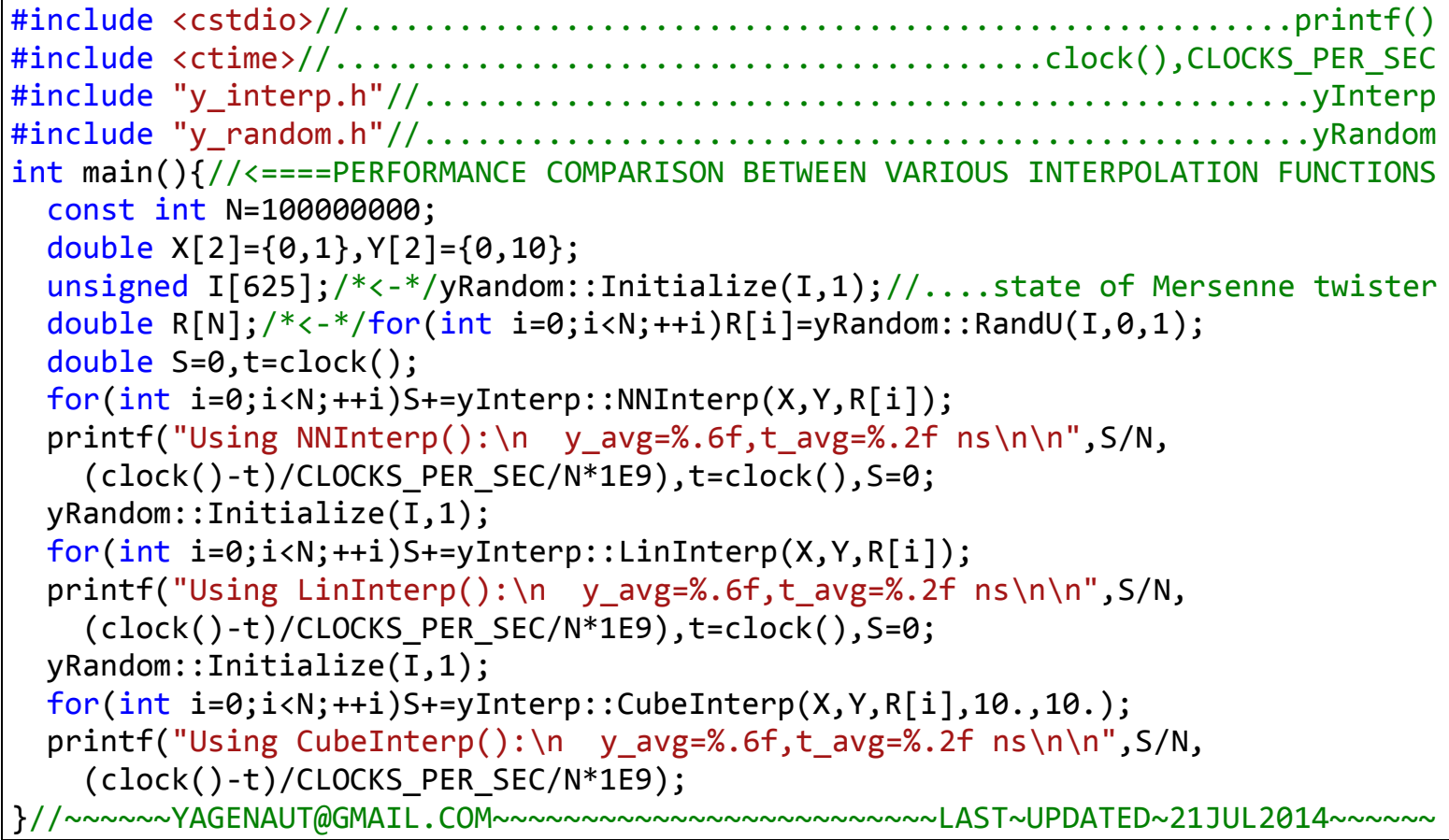

OUTPUT:

Using NNInterp():

y_avg=4.999344,t_avg=4.52 ns

Using LinInterp():

y_avg=4.999774,t_avg=2.50 ns

Using CubeInterp():

y_avg=4.999709, t_avg=3.43 ns

\section{Example: Interpolating in Two Dimensions}

The NNInterp(), LinInterp(), and CubeInterp() functions can be used to perform interpolations in 2 (and higher) dimensions.

Suppose that $z_{i, j} \equiv z\left(x_{i}, y_{j}\right)$. To interpolate in 2 dimensions, begin by performing 2 interpolations in the $\hat{y}$ direction, 1 at $x_{i}$ and 1 at $x_{i+1}$ (shown in green in Fig. 8). Define $z_{a}$ to be the interpolation at $x_{i}$ and $z_{b}$ to be the interpolation at $x_{i+1}$. Complete the interpolation by interpolating between $z_{a}$ and $z_{b}$ in the $\hat{x}$ direction (shown in red in Fig. 8). 


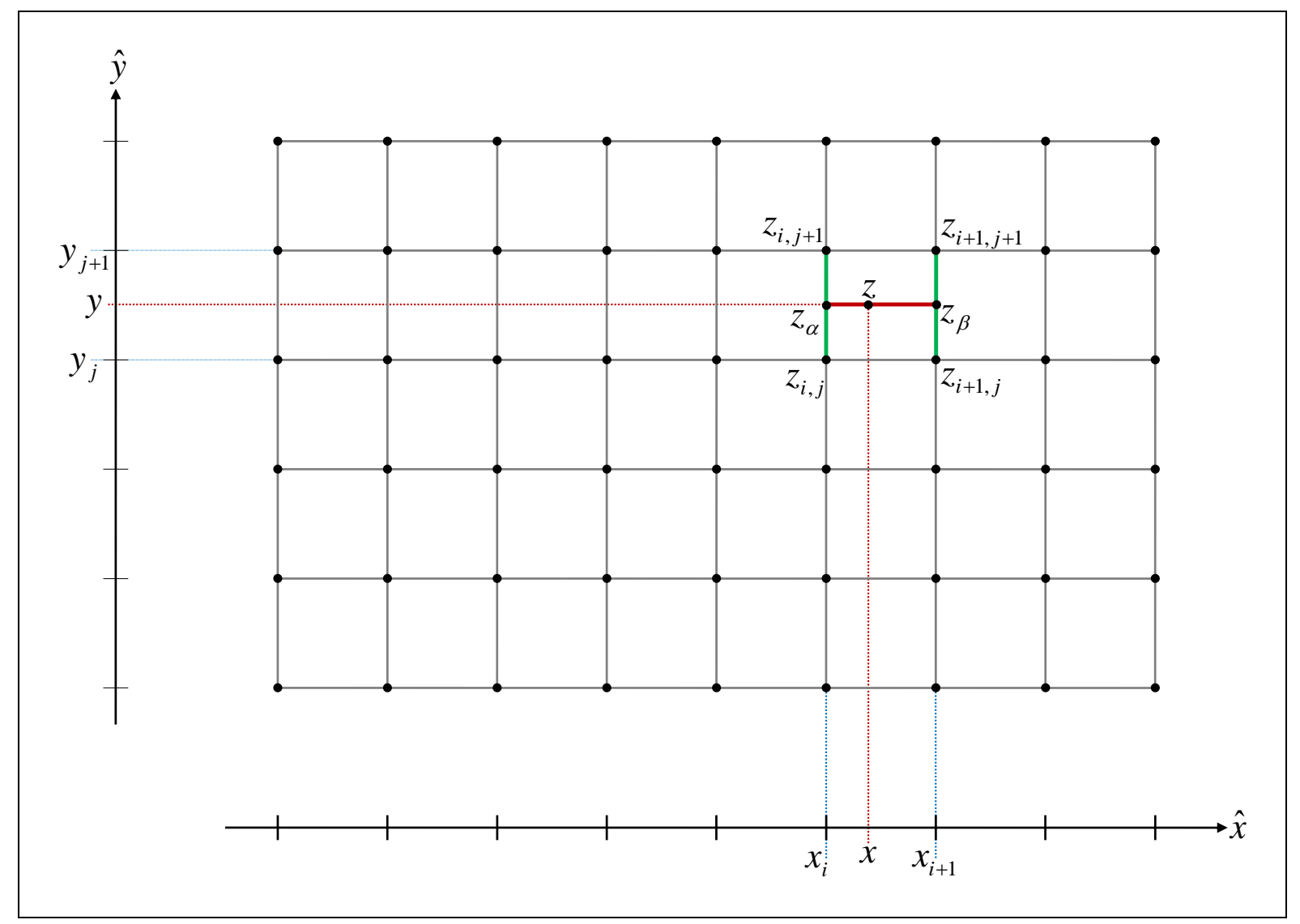

Fig. 8 Interpolating in 2 dimensions

The following example code uses the yBmp namespace to create 3 images (presented in Fig. 9) that show the results of using the NNInterp(), LinInterp(), and CubeInterp() functions to interpolate in 2 dimensions.

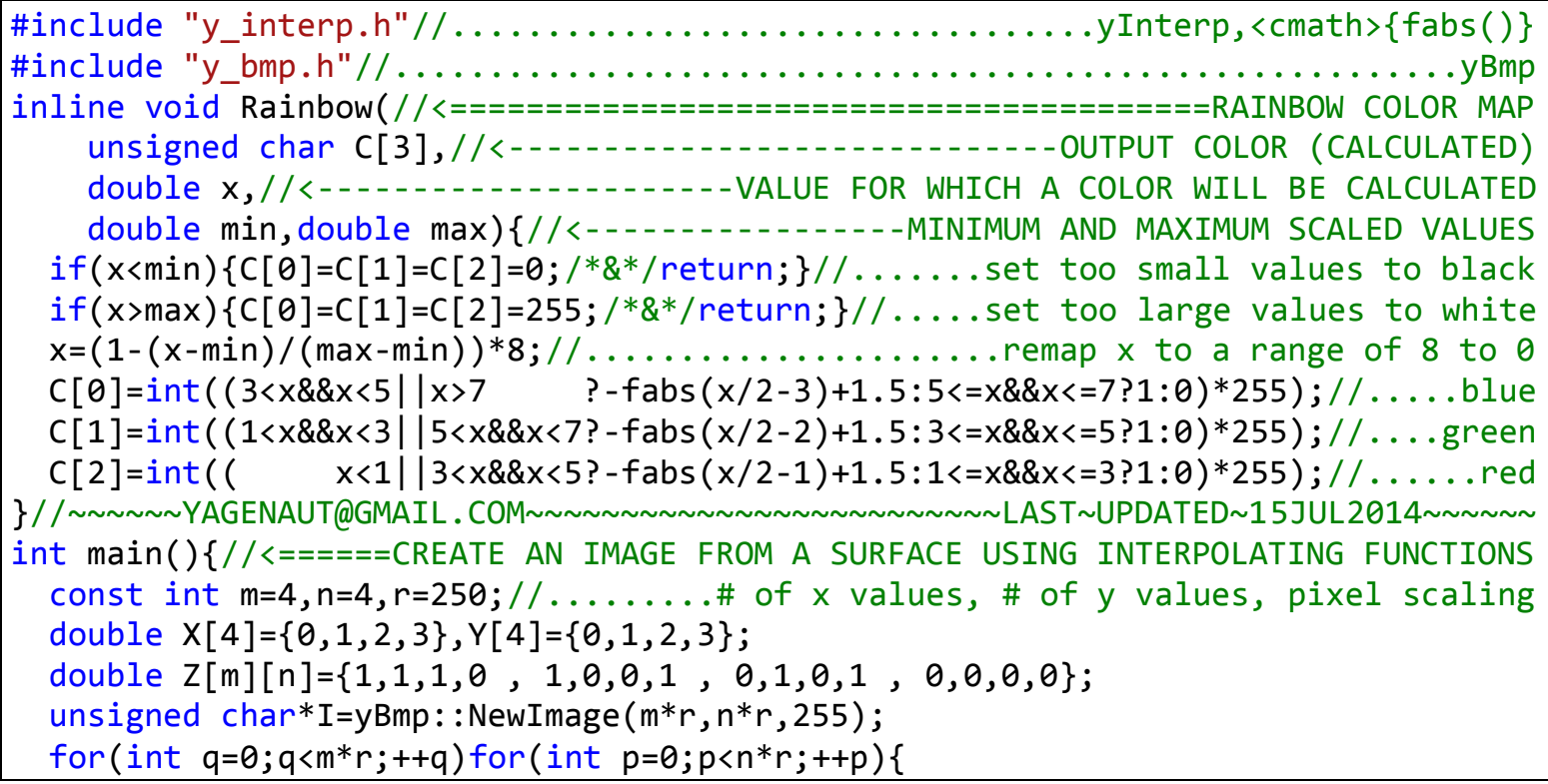



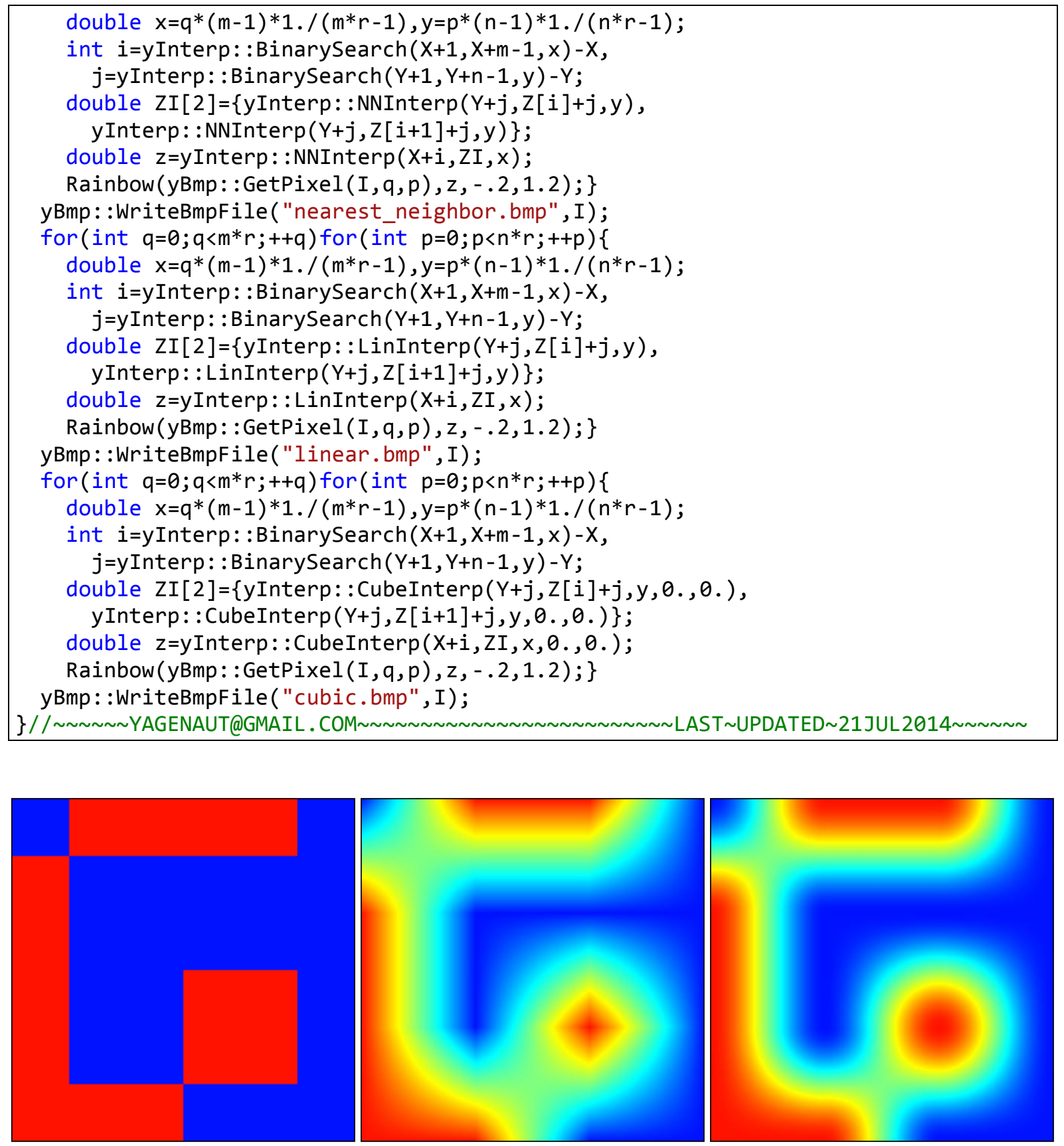

Fig. 9 2-dimensional interpolations using NNInterp() (left), LinInterp() (center), and CubeInterp() (right)

\section{Polynomial Curve Fitting: The PolyFit() Function}

Suppose that some set of $n$ ordered pairs $\left(x_{k}, y_{k}\right)$ represents a set of measurements, with $x_{k}$ being a value for an independent variable and $y_{k}$ being a value for a dependent variable. 
Furthermore, suppose that Eq. 22 represents a best-fit equation for the ordered pairs, where the coefficients $\left(c_{i}\right)$ are unknown.

$$
y(x)=c_{0}+c_{1} x+c_{2} x^{2}+\cdots+c_{i} x^{i}+\cdots+c_{d} x^{d}
$$

Eq. 23 can be used to find the coefficients for Eq. $22:^{6}$

$$
\left[\begin{array}{cccc}
n & \sum_{k=0}^{k<n} x_{k} & \ldots & \sum_{k=0}^{k<n} x_{k}^{d} \\
\sum_{k=0}^{k<n} x_{k} & \sum_{k=0}^{k<n} x_{k}^{2} & \ldots & \sum_{k=0}^{k<n} x_{k}^{d+1} \\
\vdots & \vdots & \ddots & \vdots \\
\sum_{k=0}^{k<n} x_{k}^{d} & \sum_{k=0}^{k<n} x_{k}^{d+1} & \ldots & \sum_{k=0}^{k<n} x_{k}^{2 d}
\end{array}\right]\left[\begin{array}{c}
c_{0} \\
c_{1} \\
\vdots \\
c_{d}
\end{array}\right]=\left[\begin{array}{c}
\sum_{k=0}^{k<n} y_{k} \\
k<n \\
\sum_{k=0} x_{k} y_{k} \\
\vdots \\
\sum_{k=0}^{k<n} x_{k}^{d} y_{k}
\end{array}\right]
$$

The PolyFit() function uses Gaussian elimination with backward substitution to solve Eq. 23.

\subsection{PolyFit() Code}

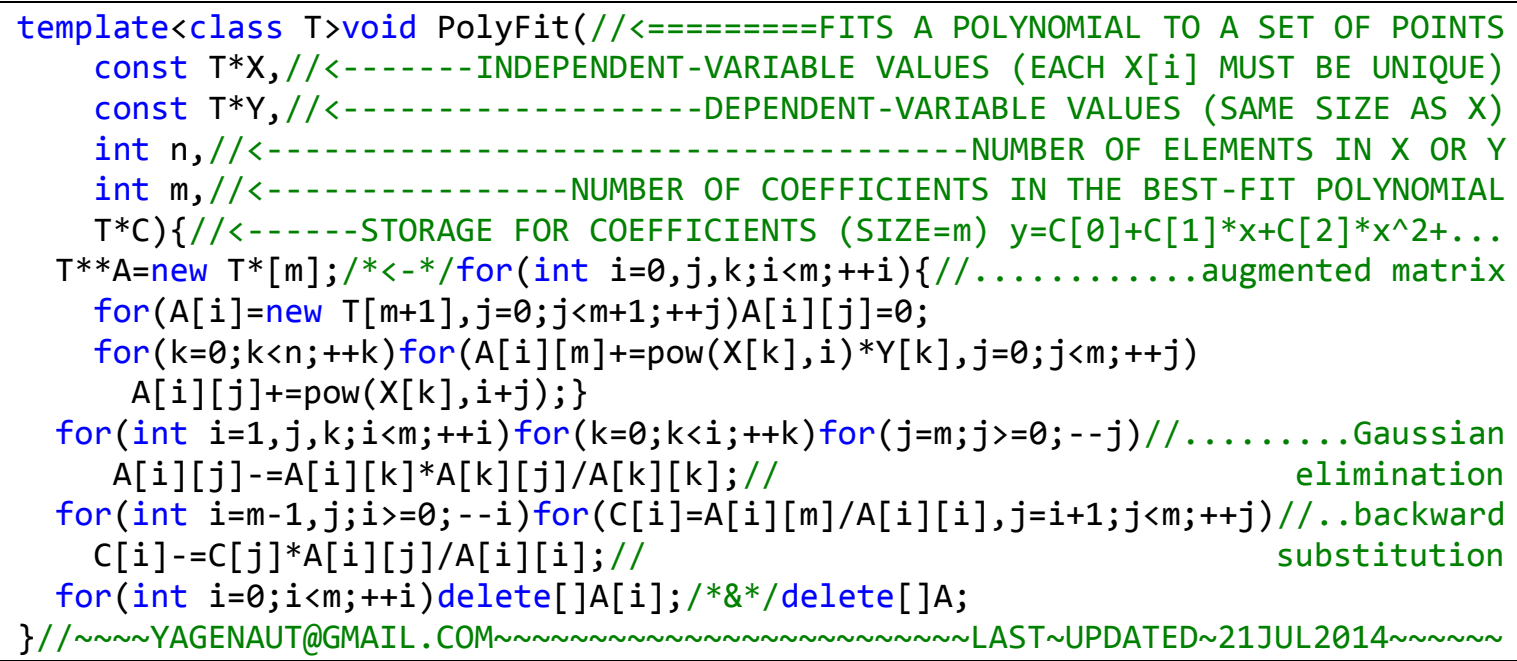

\subsection{PolyFit() Template Classes}

$\mathbf{T} \quad \mathbf{T}$ is typically a floating-point data type.

\subsection{PolyFit() Parameters}

$\mathbf{X}$

$\mathbf{Y}$

$\mathbf{n}$
$\mathbf{X}$ points to an array that is used to store the $\mathbf{n} x_{k}$ values that are specified in Eq. 23, where $\mathbf{X}=\left\{x_{0}, x_{1}, \cdots, x_{k}, \cdots, x_{n-1}\right\}$. Each $x_{k}$ value must be unique.

$\mathbf{Y}$ points to an array that is used to store the $\mathbf{n} y_{k}$ values that are specified in Eq. 23, where $\mathbf{Y}=\left\{y_{0}, y_{1}, \cdots, y_{k}, \cdots, y_{n-1}\right\}$.

n specifies $n$, the number of $x_{k}$ (or $y_{k}$ ) values. 
m

$\mathbf{m}$ specifies the number of coefficients in the array that is pointed to by $\mathbf{C}$. Thus, $\mathbf{m}=d+1$, where $d$ is the degree of the fitting polynomial (see Eq. 22).

C C points to storage for an array that is used to store the $c_{i}$ coefficients $\left(\mathbf{C}=\left\{c_{0}, c_{1}, \cdots, c_{i}, \cdots, c_{d}\right\}\right) . \mathbf{C}$ must point to an array with storage for at least $\mathbf{m}$ elements. The elements pointed to by $\mathbf{C}$ are calculated by the PolyFit() function.

Note that if $\mathbf{m}$ is too large, or if the values pointed to by $\mathbf{X}$ are too close together, then the PolyFit() function may return coefficients that do not accurately describe a best-fit curve. It is always best to plot the best-fit curve against measured data to verify the quality of the fit. It is also a good idea to calculate the coefficient of determination $\left(R^{2}\right)$, which is a measurement of the quality of the fit.

$$
R^{2} \equiv 1-\frac{\sum_{k=0}^{k<n}\left(y_{k}-f\left(x_{k}\right)\right)^{2}}{\sum_{k=0}^{k<n}\left(y_{k}-\bar{y}\right)^{2}},
$$

where

$$
\bar{y} \equiv \frac{1}{n} \sum_{k=0}^{k<n} y_{i}
$$

$R^{2}$ values are typically in the interval $[0,1]$, with 1 indicating a perfect fit.

\subsection{PolyFit() Simple Example}

The following example first defines a polynomial, then uses that polynomial to calculate a set of $\left(x_{k}, y_{k}\right)$ ordered pairs. The PolyFit() function is used to calculate the coefficients for a polynomial that best fits the set of ordered pairs. The calculated coefficients are shown to be identical (at least to 6 decimal places) to the coefficients of the original polynomial.

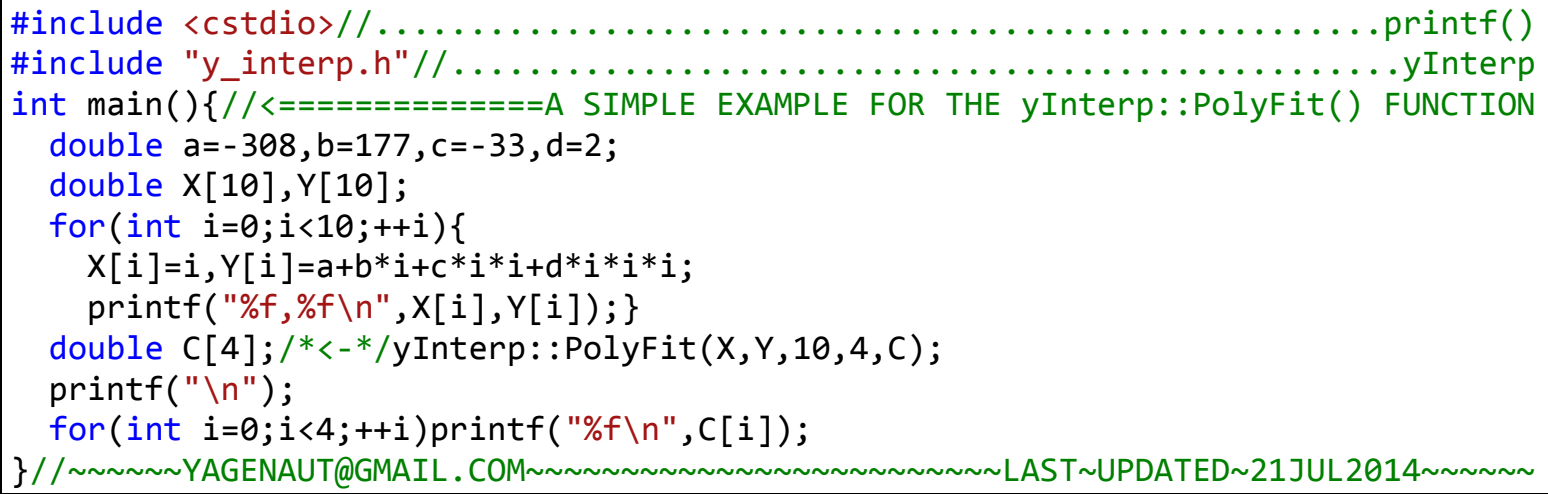




\section{OUTPUT:}

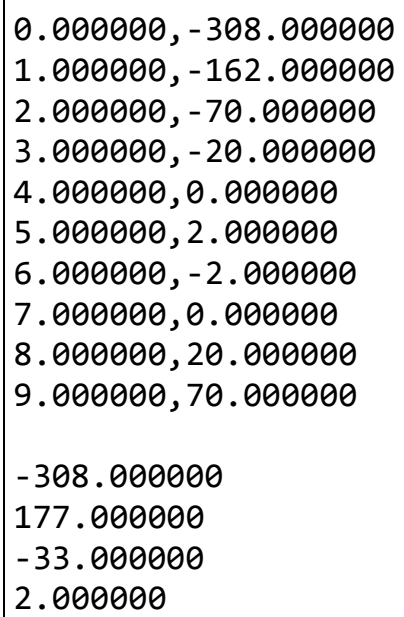

\section{Example: Exponential Fits Using the PolyFit() Function}

The PolyFit() function can be used to fit nonpolynomial equations to measured data. Suppose Eq. 26 is suspected to be a good fit for some set of data points $\left(x_{k}, y_{k}\right)$.

$$
y=A e^{B x} .
$$

The PolyFit() function can be used to find the parameters $A$ and $B$.

Begin by defining $y^{\prime}=\ln (y)$. Next, use the PolyFit() function to find $c_{0}$ and $c_{1}$ (the parameters for a straight line) for the data set $\left(x_{k}, y_{k}^{\prime}\right)$. Thus,

$$
y^{\prime}=\ln (y)=c_{0}+c_{1} x .
$$

Solving for $y$,

$$
y=e^{c_{0}+c_{1} x}=e^{c_{0}} e^{c_{1} t} .
$$

Thus,

$$
A=e^{c_{0}} \text { and } B=c_{1} \text {. }
$$

The following example code uses the PolyFit() function to fit Eq. 26 to beginning-of-year values for the Dow Jones Industrial Average for the decade 1986-1995. Values were obtained from the file “^dji_d.csv," which was downloaded from http://stooq.com. ${ }^{7}$ The yIo2 namespace was used to read and parse the data file. The results are presented in Fig. 10. 

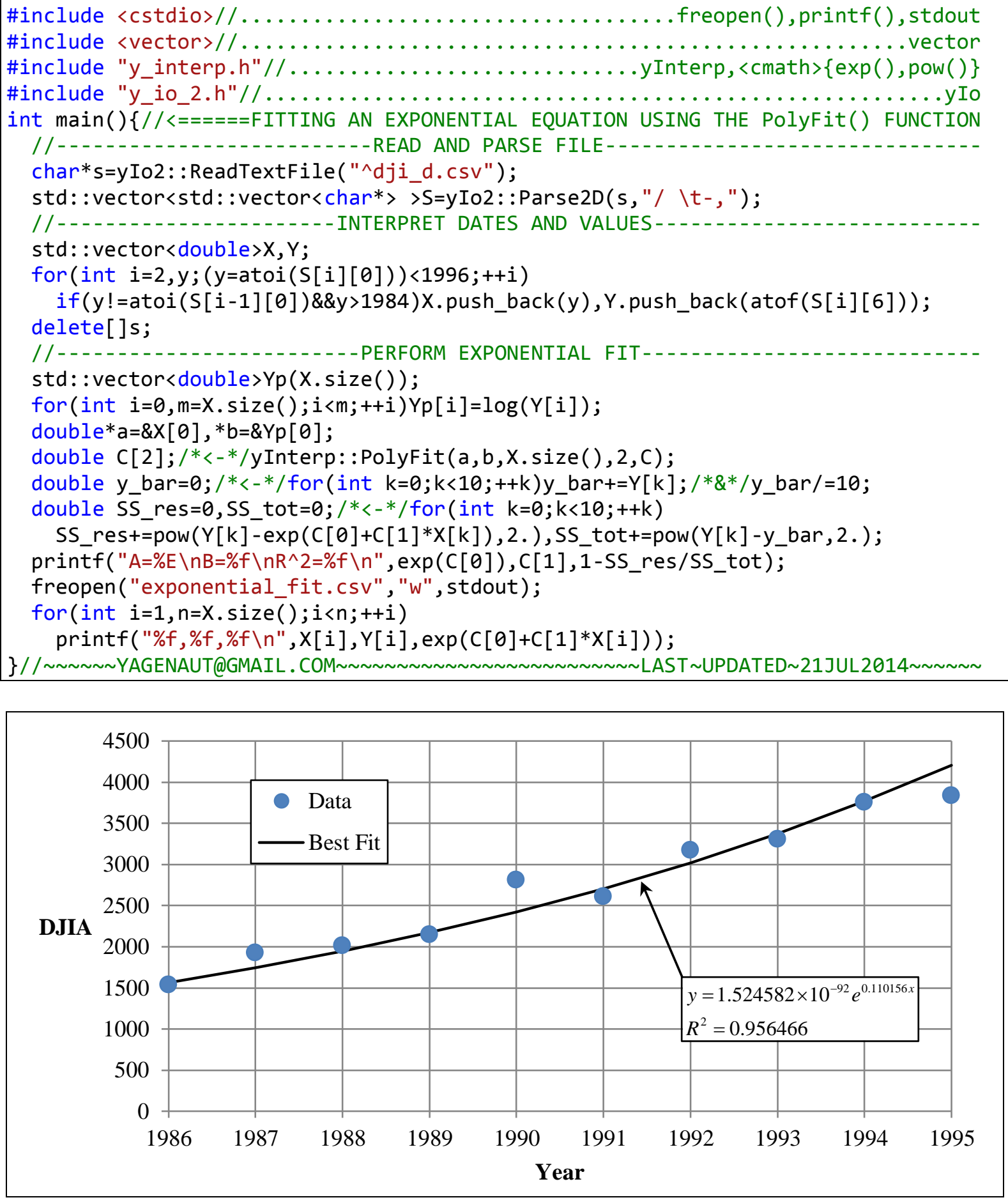

Fig. 10 Best-fit line calculated by the PolyFit() function 


\section{Code Summary}

The following summary sheet presents the yInterp namespace, which contains the BinarySearch(), PeriodicSearch(), NNInterp(), LinInterp(), CubeInterp(), CardinalSlope(), and PolyFit() functions. 


\section{yInterp Summary}

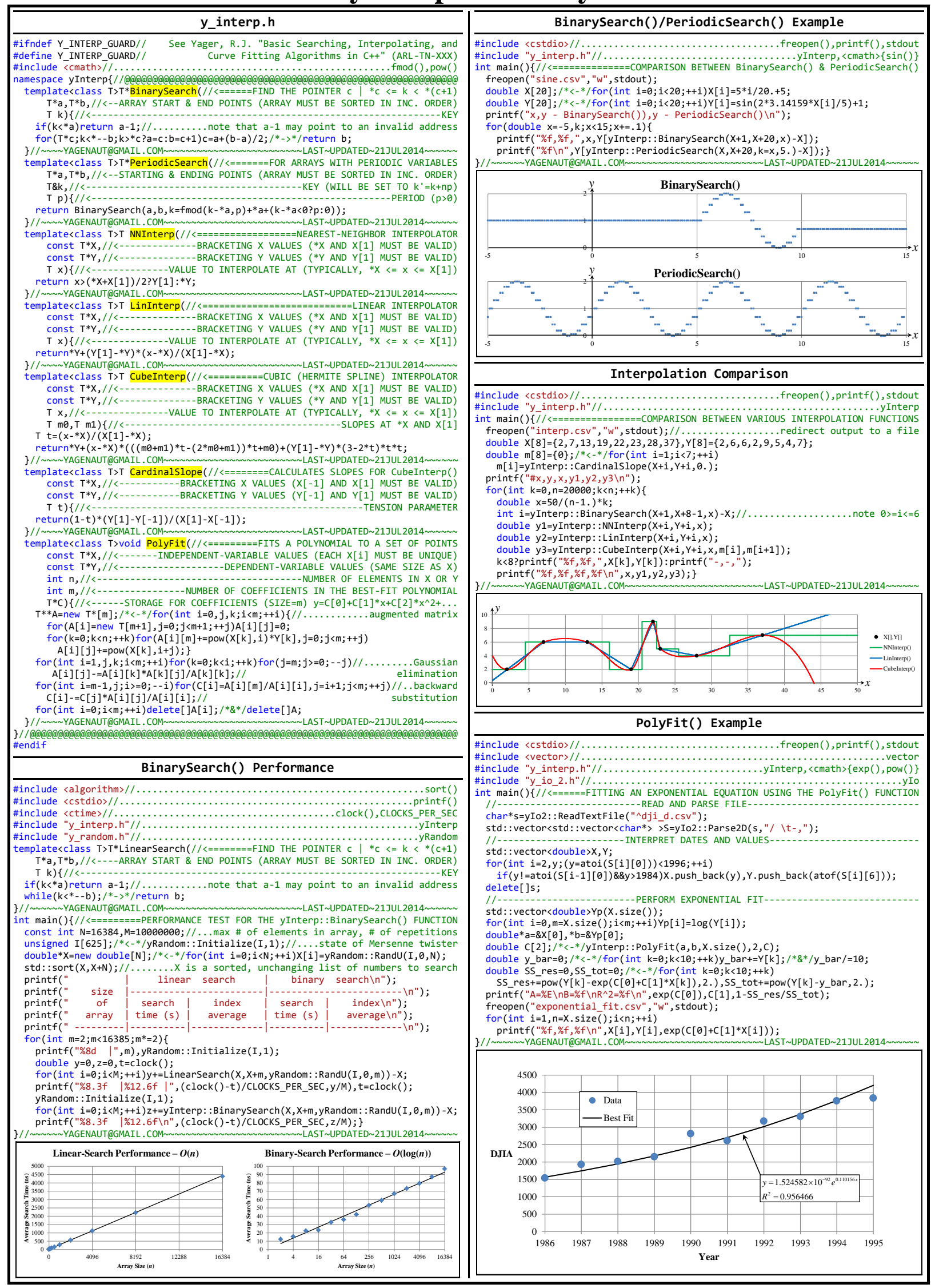




\section{References}

1. Yager RJ. Creating, Searching, and Deleting KD Trees Using C++. Aberdeen Proving Ground (MD): Army Research Laboratory (US). September 2014. Report No.: ARL-TN0629.

2. Yager RJ. Working With Evenly Spaced, Rectangular Surface Grids Using C++. Aberdeen Proving Ground (MD): Army Research Laboratory (US). October 2014. Report No.: ARLTN-0641.

3. Yager RJ. Generating Pseudorandom Numbers from Various Distributions Using C++. U.S. Army Research Laboratory: Aberdeen Proving Ground, MD, June 2014. Report No.: ARLTN-613.

4. Yager RJ. Reading, Writing, and Modifying BMP Files Using C++. Aberdeen Proving Ground (MD): Army Research Laboratory (US); August 2013. Report No.: ARL-TN-559.

5. Yager RJ. Reading, Writing, and Parsing Text Files Using C++ (Updated). Aberdeen Proving Ground (MD): Army Research Laboratory (US). October 2014. Report No.: ARLTN-0642.

6. Lancaster P, Salkauskas K. Curve and Surface Fitting, an Introduction. San Diego (CA): Academic Press Ltd, 1987.

7. “^dji_d.csv”. [accessed 2014 Jun 19] http://stooq.com/q/d/?s=^dji. 


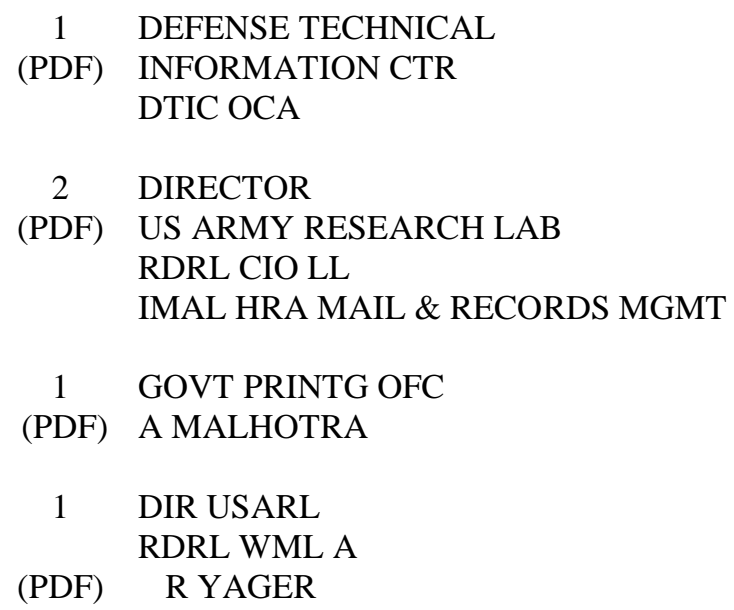


INTENTIONALLY LEFT BLANK. 